\title{
Drinfeld twists of the open XXZ chain with non-diagonal boundary terms
}

\author{
Wen-Li Yang $^{a}$ and Yao-Zhong Zhang ${ }^{b}$ \\ ${ }^{a}$ Institute of Modern Physics, Northwest University, Xian 710069, P.R. China \\ ${ }^{b}$ The University of Queensland, School of Mathematics and Physics, Brisbane, QLD 4072, \\ Australia \\ E-mail: wlyang@nwu.edu.cn, yzz@maths.uq.edu.au
}

\begin{abstract}
The Drinfeld twists or factorizing F-matrices for the open XXZ spin chain with non-diagonal boundary terms are constructed. It is shown that in the F-basis the two sets of pseudo-particle creation operators simultaneously take completely symmetric and polarization free form. The explicit and completely symmetric expressions of the two sets of Bethe states of the model are obtained.
\end{abstract}

PACS: 03.65.Fd; 04.20.Jb; 05.30.-d; 75.10.Jm

Keywords: The open XXZ chain; Algebraic Bethe ansatz; Drinfeld twist. 


\section{Introduction}

The algebraic Bethe ansatz [1] has been proven to provide a powerful tool of solving eigenvalue problems of quantum integrable systems such as quantum spin chains. In this framework, the pseudo-particle creation and annihilation operators are constructed by off-diagonal matrix elements of the so-called monodromy matrix. The Bethe states (eigenstates) of transfer matrix are obtained by applying creation operators to the reference state (or pseudovacuum state). However, the apparently simple action of creation operators is plagued with non-local effects arising from polarization clouds or compensating exchange terms on the level of local operators. This make the explicit construction of the Bethe states challenging.

In 2], Maillet and Sanchez de Santos showed how monodromy matrices of the inhomogeneous XXX and XXZ spin chains with periodic boundary conditions (or the closed XXX and XXZ chains) can be simplified by using the Drinfeld twists or factorizing F-matrices [3]. This leads to the so-called F-basis provided by the F-matrix for the analysis of these models. In the F-basis, the pseudo-particle creation and annihilation operators of the models take completely symmetric forms and contain no compensating exchange terms on the level of local operators (i.e. polarization free). As a result, the Bethe states of the models are simplified dramatically and can be written down explicitly [4. Similar results have been obtained for other models with periodic boundary conditions [5, 6, 7, 8, 9, 10,

Recently, it was shown [11, 12] that the F-matrices of the closed XXX and XXZ chains also make the pseudo-particle creation operators of the open XXX and XXZ chains with diagonal boundary terms polarization free. This is mainly due to the fact that the closed chain and the corresponding open chain with diagonal boundary terms share the same reference state [13]. However, the story for the open XXZ chain with non-diagonal boundary terms is quite different [14, 15, 16, 17, 18, 19, 20, 21, 22, 223, 24, 25, 26, 27]. Firstly, the reference state (all spin up state) of the closed chain is no longer a reference state of the open chain with non-diagonal boundary terms [15, 16, 20]. Secondly, two reference states (and thus two sets of Bethe states) are needed [28] for the open XXZ chain with non-diagonal boundary terms in order to obtain its complete spectrum [29, 30]. As a consequence, the F-matrix found in

[2] is no longer the desirable F-matrix for the open XXZ chain with non-diagonal boundary terms.

In this paper, we obtain the Drinfeld twists or factorizing F-matrices for the open XXZ 
chain with integrable boundary conditions given by the non-diagonal K-matrices (2.9) and (2.11). We find that in the F-basis the two sets of pseudo-particle creation operators (acting on the two reference states) of the boundary model simultaneously take completely symmetric and polarization free forms. These enable us to derive the explicit and completely symmetric expressions of the two sets of Bethe states of the model.

The paper is organized as follows. In section 2, we briefly describe the open XXZ chain with non-diagonal boundary terms and introduce the pseudo-particle creation operators and the two sets of Bethe states of the model. In section 3, we introduce a new pseudo-particle creation operator. This operator is used to generate a new set of Bethe states. Section 4 introduces the one-row and double row monodromy matrices in the face picture for the open XXZ chain. In section 5, we construct the F-matrix of the open XXZ chain in the face picture and obtain the completely symmetric and polarization free representations of the pseudo-particle creation operators and the complete symmetric expressions of the two sets of Bethe states in the F-basis. In section 6, we summarize our results and give some discussions. Some details about the new set of Bethe states are given in Appendix A.

\section{The inhomogeneous spin- $\frac{1}{2} \mathrm{XXZ}$ open chain}

Throughout, $V$ denotes a two-dimensional linear space. The spin- $\frac{1}{2} \mathrm{XXZ}$ chain can be constructed from the well-known six-vertex model R-matrix $\bar{R}(u) \in \operatorname{End}(V \otimes V)[1$ given by

$$
\bar{R}(u)=\left(\begin{array}{llll}
1 & & & \\
& b(u) & c(u) & \\
& c(u) & b(u) & \\
& & & 1
\end{array}\right) .
$$

The coefficient functions read: $b(u)=\frac{\sin u}{\sin (u+\eta)}, c(u)=\frac{\sin \eta}{\sin (u+\eta)}$. Here we assume $\eta$ is a generic complex number. The R-matrix satisfies the quantum Yang-Baxter equation (QYBE),

$$
R_{1,2}\left(u_{1}-u_{2}\right) R_{1,3}\left(u_{1}-u_{3}\right) R_{2,3}\left(u_{2}-u_{3}\right)=R_{2,3}\left(u_{2}-u_{3}\right) R_{1,3}\left(u_{1}-u_{3}\right) R_{1,2}\left(u_{1}-u_{2}\right),
$$

and the unitarity, crossing-unitarity and quasi-classical properties [20]. We adopt the standard notations: for any matrix $A \in \operatorname{End}(V), A_{j}$ (or $A^{j}$ ) is an embedding operator in the tensor space $V \otimes V \otimes \cdots$, which acts as $A$ on the $j$-th space and as identity on the other factor spaces; $R_{i, j}(u)$ is an embedding operator of R-matrix in the tensor space, which acts as identity on the factor spaces except for the $i$-th and $j$-th ones. 
One introduces the "row-to-row" (or one-row ) monodromy matrix $T(u)$, which is an $2 \times 2$ matrix with elements being operators acting on $V^{\otimes N}$, where $N=2 M$ ( $M$ being a positive integer),

$$
T_{0}(u)=\bar{R}_{0, N}\left(u-z_{N}\right) \bar{R}_{0, N-1}\left(u-z_{N-1}\right) \cdots \bar{R}_{0,1}\left(u-z_{1}\right) .
$$

Here $\left\{z_{j} \mid j=1, \cdots, N\right\}$ are arbitrary free complex parameters which are usually called inhomogeneous parameters.

Integrable open chain can be constructed as follows [13]. Let us introduce a pair of K-matrices $K^{-}(u)$ and $K^{+}(u)$. The former satisfies the reflection equation (RE)

$$
\begin{aligned}
& \bar{R}_{1,2}\left(u_{1}-u_{2}\right) K_{1}^{-}\left(u_{1}\right) \bar{R}_{2,1}\left(u_{1}+u_{2}\right) K_{2}^{-}\left(u_{2}\right) \\
& =K_{2}^{-}\left(u_{2}\right) \bar{R}_{1,2}\left(u_{1}+u_{2}\right) K_{1}^{-}\left(u_{1}\right) \bar{R}_{2,1}\left(u_{1}-u_{2}\right),
\end{aligned}
$$

and the latter satisfies the dual RE

$$
\begin{aligned}
& \bar{R}_{1,2}\left(u_{2}-u_{1}\right) K_{1}^{+}\left(u_{1}\right) \bar{R}_{2,1}\left(-u_{1}-u_{2}-2 \eta\right) K_{2}^{+}\left(u_{2}\right) \\
& \quad=K_{2}^{+}\left(u_{2}\right) \bar{R}_{1,2}\left(-u_{1}-u_{2}-2 \eta\right) K_{1}^{+}\left(u_{1}\right) \bar{R}_{2,1}\left(u_{2}-u_{1}\right) .
\end{aligned}
$$

For open spin-chains, instead of the standard "row-to-row" monodromy matrix $T(u)(2.3)$, one needs to consider the "double-row" monodromy matrix $\mathbb{T}(u)$

$$
\mathbb{T}(u)=T(u) K^{-}(u) \hat{T}(u), \quad \hat{T}(u)=T^{-1}(-u) .
$$

Then the double-row transfer matrix of the XXZ chain with open boundary (or the open $\mathrm{XXZ}$ chain) is given by

$$
\tau(u)=\operatorname{tr}\left(K^{+}(u) \mathbb{T}(u)\right)
$$

The QYBE and (dual) REs lead to that the transfer matrices with different spectral parameters commute with each other $[13]:[\tau(u), \tau(v)]=0$. This ensures the integrability of the open XXZ chain.

In this paper, we will consider the K-matrix $K^{-}(u)$ which is a generic solution to the RE (2.4) associated the six-vertex model R-matrix [31, 32]

$$
K^{-}(u)=\left(\begin{array}{cc}
k_{1}^{1}(u) & k_{2}^{1}(u) \\
k_{1}^{2}(u) & k_{2}^{2}(u)
\end{array}\right) \equiv K(u)
$$


The coefficient functions are

$$
\begin{aligned}
k_{1}^{1}(u) & =\frac{\cos \left(\lambda_{1}-\lambda_{2}\right)-\cos \left(\lambda_{1}+\lambda_{2}+2 \xi\right) e^{-2 i u}}{2 \sin \left(\lambda_{1}+\xi+u\right) \sin \left(\lambda_{2}+\xi+u\right)}, \\
k_{2}^{1}(u) & =\frac{-i \sin (2 u) e^{-i\left(\lambda_{1}+\lambda_{2}\right)} e^{-i u}}{2 \sin \left(\lambda_{1}+\xi+u\right) \sin \left(\lambda_{2}+\xi+u\right)}, \\
k_{1}^{2}(u) & =\frac{i \sin (2 u) e^{i\left(\lambda_{1}+\lambda_{2}\right)} e^{-i u}}{2 \sin \left(\lambda_{1}+\xi+u\right) \sin \left(\lambda_{2}+\xi+u\right)}, \\
k_{2}^{2}(u) & =\frac{\cos \left(\lambda_{1}-\lambda_{2}\right) e^{-2 i u}-\cos \left(\lambda_{1}+\lambda_{2}+2 \xi\right)}{2 \sin \left(\lambda_{1}+\xi+u\right) \sin \left(\lambda_{2}+\xi+u\right)} .
\end{aligned}
$$

At the same time, we introduce the corresponding dual K-matrix $K^{+}(u)$ which is a generic solution to the dual reflection equation (2.5) with a particular choice of the free boundary parameters:

$$
K^{+}(u)=\left(\begin{array}{cc}
k_{1}^{+1}(u) & k_{2}^{+1}(u) \\
k_{1}^{+2}(u) & k_{2}^{+2}(u)
\end{array}\right)
$$

with the matrix elements

$$
\begin{aligned}
k_{1}^{+1}(u) & =\frac{\cos \left(\lambda_{1}-\lambda_{2}\right) e^{-i \eta}-\cos \left(\lambda_{1}+\lambda_{2}+2 \bar{\xi}\right) e^{2 i u+i \eta}}{2 \sin \left(\lambda_{1}+\bar{\xi}-u-\eta\right) \sin \left(\lambda_{2}+\bar{\xi}-u-\eta\right)}, \\
k^{+1}(u) & =\frac{i \sin (2 u+2 \eta) e^{-i\left(\lambda_{1}+\lambda_{2}\right)} e^{i u-i \eta}}{2 \sin \left(\lambda_{1}+\bar{\xi}-u-\eta\right) \sin \left(\lambda_{2}+\bar{\xi}-u-\eta\right)}, \\
k^{+2}(u) & =\frac{-i \sin (2 u+2 \eta) e^{i\left(\lambda_{1}+\lambda_{2}\right)} e^{i u+i \eta}}{2 \sin \left(\lambda_{1}+\bar{\xi}-u-\eta\right) \sin \left(\lambda_{2}+\bar{\xi}-u-\eta\right)}, \\
k^{+2}(u) & =\frac{\cos \left(\lambda_{1}-\lambda_{2}\right) e^{2 i u+i \eta}-\cos \left(\lambda_{1}+\lambda_{2}+2 \bar{\xi}\right) e^{-i \eta}}{2 \sin \left(\lambda_{1}+\bar{\xi}-u-\eta\right) \sin \left(\lambda_{2}+\bar{\xi}-u-\eta\right)} .
\end{aligned}
$$

The K-matrices depend on four free boundary parameters $\left\{\lambda_{1}, \lambda_{2}, \xi, \bar{\xi}\right\} 11$. It is very convenient to introduce a vector $\lambda \in V$ associated with the boundary parameters $\left\{\lambda_{i}\right\}$,

$$
\lambda=\sum_{k=1}^{2} \lambda_{k} \epsilon_{k},
$$

where $\left\{\epsilon_{i}, i=1,2\right\}$ form the orthonormal basis of $V$ such that $\left\langle\epsilon_{i}, \epsilon_{j}\right\rangle=\delta_{i j}$.

\subsection{Vertex-face correspondence}

Let us briefly review the face-type R-matrix associated with the six-vertex model.

\footnotetext{
${ }^{1}$ To our knowledge, this is the only case for which the complete eigenstates of the open XXZ chain with non-diagonal boundary terms can be constructed through the algebraic Bethe ansatz method [28].
} 
Set

$$
\hat{\imath}=\epsilon_{i}-\bar{\epsilon}, \quad \bar{\epsilon}=\frac{1}{2} \sum_{k=1}^{2} \epsilon_{k}, \quad i=1,2, \quad \text { then } \sum_{i=1}^{2} \hat{\imath}=0 .
$$

Let $\mathfrak{h}$ be the Cartan subalgebra of $A_{1}$ and $\mathfrak{h}^{*}$ be its dual. A finite dimensional diagonalizable $\mathfrak{h}$-module is a complex finite dimensional vector space $W$ with a weight decomposition $W=$ $\oplus_{\mu \in \mathfrak{h}^{*}} W[\mu]$, so that $\mathfrak{h}$ acts on $W[\mu]$ by $x v=\mu(x) v,(x \in \mathfrak{h}, v \in W[\mu])$. For example, the non-zero weight spaces of the fundamental representation $V_{\Lambda_{1}}=\mathbb{C}^{2}=V$ are

$$
W[\hat{\imath}]=\mathbb{C} \epsilon_{i}, \quad i=1,2 .
$$

For a generic $m \in V$, define

$$
m_{i}=\left\langle m, \epsilon_{i}\right\rangle, \quad m_{i j}=m_{i}-m_{j}=\left\langle m, \epsilon_{i}-\epsilon_{j}\right\rangle, \quad i, j=1,2 .
$$

Let $R(u, m) \in \operatorname{End}(V \otimes V)$ be the R-matrix of the six-vertex SOS model, which is trigonometric limit of the eight-vertex SOS model [33] given by

$$
R(u ; m)=\sum_{i=1}^{2} R(u ; m)_{i i}^{i i} E_{i i} \otimes E_{i i}+\sum_{i \neq j}^{2}\left\{R(u ; m)_{i j}^{i j} E_{i i} \otimes E_{j j}+R(u ; m)_{i j}^{j i} E_{j i} \otimes E_{i j}\right\}
$$

where $E_{i j}$ is the matrix with elements $\left(E_{i j}\right)_{k}^{l}=\delta_{j k} \delta_{i l}$. The coefficient functions are

$$
\begin{aligned}
& R(u ; m)_{i i}^{i i}=1, \quad R(u ; m)_{i j}^{i j}=\frac{\sin u \sin \left(m_{i j}-\eta\right)}{\sin (u+\eta) \sin \left(m_{i j}\right)}, \quad i \neq j, \\
& R(u ; m)_{i j}^{j i}=\frac{\sin \eta \sin \left(u+m_{i j}\right)}{\sin (u+\eta) \sin \left(m_{i j}\right)}, \quad i \neq j,
\end{aligned}
$$

and $m_{i j}$ is defined in (2.15). The R-matrix satisfies the dynamical (modified) quantum Yang-Baxter equation (or the star-triangle relation) [33]

$$
\begin{array}{r}
R_{1,2}\left(u_{1}-u_{2} ; m-\eta h^{(3)}\right) R_{1,3}\left(u_{1}-u_{3} ; m\right) R_{2,3}\left(u_{2}-u_{3} ; m-\eta h^{(1)}\right) \\
=R_{2,3}\left(u_{2}-u_{3} ; m\right) R_{1,3}\left(u_{1}-u_{3} ; m-\eta h^{(2)}\right) R_{1,2}\left(u_{1}-u_{2} ; m\right) .
\end{array}
$$

Here we have adopted

$$
R_{1,2}\left(u, m-\eta h^{(3)}\right) v_{1} \otimes v_{2} \otimes v_{3}=(R(u, m-\eta \mu) \otimes \mathrm{id}) v_{1} \otimes v_{2} \otimes v_{3}, \quad \text { if } v_{3} \in W[\mu] .
$$

Moreover, one may check that the R-matrix satisfies weight conservation condition,

$$
\left[h^{(1)}+h^{(2)}, R_{1,2}(u ; m)\right]=0,
$$


unitary condition,

$$
R_{1,2}(u ; m) R_{2,1}(-u ; m)=\mathrm{id} \otimes \mathrm{id}
$$

and crossing relation

$$
R(u ; m)_{i j}^{k l}=\varepsilon_{l} \varepsilon_{j} \frac{\sin (u) \sin \left((m-\eta \hat{\imath})_{21}\right)}{\sin (u+\eta) \sin \left(m_{21}\right)} R(-u-\eta ; m-\eta \hat{\imath})_{\bar{l} i}^{\bar{j} k},
$$

where

$$
\varepsilon_{1}=1, \varepsilon_{2}=-1, \quad \text { and } \overline{1}=2, \overline{2}=1 \text {. }
$$

Define the following functions: $\theta^{(1)}(u)=e^{-i u}, \theta^{(2)}(u)=1$. Let us introduce two intertwiners which are 2-component column vectors $\phi_{m, m-\eta \hat{\jmath}}(u)$ labelled by $\hat{1}, \hat{2}$. The $k$-th element of $\phi_{m, m-\eta \hat{\jmath}}(u)$ is given by

$$
\phi_{m, m-\eta \hat{\jmath}}^{(k)}(u)=\theta^{(k)}\left(u+2 m_{j}\right) .
$$

Explicitly,

$$
\phi_{m, m-\eta \hat{1}}(u)=\left(\begin{array}{c}
e^{-i\left(u+2 m_{1}\right)} \\
1
\end{array}\right), \quad \phi_{m, m-\eta \hat{2}}(u)=\left(\begin{array}{c}
e^{-i\left(u+2 m_{2}\right)} \\
1
\end{array}\right) .
$$

Obviously, the two intertwiner vectors $\phi_{m, m-\eta \hat{\imath}}(u)$ are linearly independent for a generic $m \in V$.

Using the intertwiner vectors, one can derive the following face-vertex correspondence relation [15]

$$
\begin{aligned}
\bar{R}_{1,2}( & \left.u_{1}-u_{2}\right) \phi_{m, m-\eta \hat{\imath}}^{1}\left(u_{1}\right) \phi_{m-\eta \hat{\imath}, m-\eta(\hat{\imath}+\hat{\jmath})}^{2}\left(u_{2}\right) \\
& =\sum_{k, l} R\left(u_{1}-u_{2} ; m\right)_{i j}^{k l} \phi_{m-\eta \hat{l}, m-\eta(\hat{l}+\hat{k})}^{1}\left(u_{1}\right) \phi_{m, m-\eta \hat{l}}^{2}\left(u_{2}\right) .
\end{aligned}
$$

Then the QYBE (2.2) of the vertex-type R-matrix $\bar{R}(u)$ is equivalent to the dynamical YangBaxter equation (2.19) of the SOS R-matrix $R(u, m)$. For a generic $m$, we can introduce other types of intertwiners $\bar{\phi}, \tilde{\phi}$ which are both row vectors and satisfy the following conditions,

$$
\bar{\phi}_{m, m-\eta \hat{\mu}}(u) \phi_{m, m-\eta \hat{\nu}}(u)=\delta_{\mu \nu}, \quad \tilde{\phi}_{m+\eta \hat{\mu}, m}(u) \phi_{m+\eta \hat{\nu}, m}(u)=\delta_{\mu \nu}
$$

from which one can derive the relations,

$$
\begin{aligned}
& \sum_{\mu=1}^{2} \phi_{m, m-\eta \hat{\mu}}(u) \bar{\phi}_{m, m-\eta \hat{\mu}}(u)=\mathrm{id} \\
& \sum_{\mu=1}^{2} \phi_{m+\eta \hat{\mu}, m}(u) \tilde{\phi}_{m+\eta \hat{\mu}, m}(u)=\mathrm{id} .
\end{aligned}
$$


With the help of (2.27)-(2.30), we obtain,

$$
\begin{aligned}
& \tilde{\phi}_{m+\eta \hat{k}, m}^{1}\left(u_{1}\right) \bar{R}_{1,2}\left(u_{1}-u_{2}\right) \phi_{m+\eta \hat{\jmath}, m}^{2}\left(u_{2}\right) \\
& =\sum_{i, l} R\left(u_{1}-u_{2} ; m\right)_{i j}^{k l} \tilde{\phi}_{m+\eta(\hat{\imath}+\hat{\jmath}), m+\eta \hat{\jmath}}^{1}\left(u_{1}\right) \phi_{m+\eta(\hat{k}+\hat{l}), m+\eta \hat{k}}^{2}\left(u_{2}\right), \\
& \tilde{\phi}_{m+\eta \hat{k}, m}^{1}\left(u_{1}\right) \tilde{\phi}_{m+\eta(\hat{k}+\hat{l}), m+\eta \hat{k}}^{2}\left(u_{2}\right) \bar{R}_{1,2}\left(u_{1}-u_{2}\right) \\
& =\sum_{i, j} R\left(u_{1}-u_{2} ; m\right)_{i j}^{k l} \tilde{\phi}_{m+\eta(\hat{\imath}+\hat{\jmath}), m+\eta \hat{\jmath}}^{1}\left(u_{1}\right) \tilde{\phi}_{m+\eta \hat{\jmath}, m}^{2}\left(u_{2}\right), \\
& \bar{\phi}_{m, m-\eta \hat{l}}^{2}\left(u_{2}\right) \bar{R}_{1,2}\left(u_{1}-u_{2}\right) \phi_{m, m-\eta \hat{\imath}}^{1}\left(u_{1}\right) \\
& =\sum_{k, j} R\left(u_{1}-u_{2} ; m\right)_{i j}^{k l} \phi_{m-\eta \hat{l}, m-\eta(\hat{k}+\hat{l})}^{1}\left(u_{1}\right) \bar{\phi}_{m-\eta \hat{\imath}, m-\eta(\hat{\imath}+\hat{\jmath})}^{2}\left(u_{2}\right), \\
& \bar{\phi}_{m-\eta \hat{l}, m-\eta(\hat{k}+\hat{l})}^{1}\left(u_{1}\right) \bar{\phi}_{m, m-\eta \hat{l}}^{2}\left(u_{2}\right) \bar{R}_{12}\left(u_{1}-u_{2}\right) \\
& =\sum_{i, j} R\left(u_{1}-u_{2} ; m\right)_{i j}^{k l} \bar{\phi}_{m, m-\eta \hat{\imath}}^{1}\left(u_{1}\right) \bar{\phi}_{m-\eta \hat{\imath}, m-\eta(\hat{\imath}+\hat{\jmath})}^{2}\left(u_{2}\right) .
\end{aligned}
$$

One may verify that the K-matrices $K^{ \pm}(u)$ given by (2.8) and (2.10) can be expressed in terms of the intertwiners and diagonal matrices $\mathcal{K}(\lambda \mid u)$ and $\tilde{\mathcal{K}}(\lambda \mid u)$ as follows

$$
\begin{aligned}
K^{-}(u)_{t}^{s} & =\sum_{i, j} \phi_{\lambda-\eta(\hat{\imath}-\hat{\jmath}), \lambda-\eta \hat{\imath}}^{(s)}(u) \mathcal{K}(\lambda \mid u)_{i}^{j} \bar{\phi}_{\lambda, \lambda-\eta \hat{\imath}}^{(t)}(-u), \\
K^{+}(u)_{t}^{s} & =\sum_{i, j} \phi_{\lambda, \lambda-\eta \hat{\jmath}}^{(s)}(-u) \tilde{\mathcal{K}}(\lambda \mid u)_{i}^{j} \tilde{\phi}_{\lambda-\eta(\hat{\jmath}-\hat{\imath}), \lambda-\eta \hat{\jmath}}^{(t)}(u) .
\end{aligned}
$$

Here the two diagonal matrices $\mathcal{K}(\lambda \mid u)$ and $\tilde{\mathcal{K}}(\lambda \mid u)$ are given by

$$
\begin{aligned}
\mathcal{K}(\lambda \mid u) & \equiv \operatorname{Diag}\left(k(\lambda \mid u)_{1}, k(\lambda \mid u)_{2}\right)=\operatorname{Diag}\left(\frac{\sin \left(\lambda_{1}+\xi-u\right)}{\sin \left(\lambda_{1}+\xi+u\right)}, \frac{\sin \left(\lambda_{2}+\xi-u\right)}{\sin \left(\lambda_{2}+\xi+u\right)}\right), \\
\tilde{\mathcal{K}}(\lambda \mid u) & \equiv \operatorname{Diag}\left(\tilde{k}(\lambda \mid u)_{1}, \tilde{k}(\lambda \mid u)_{2}\right) \\
& =\operatorname{Diag}\left(\frac{\sin \left(\lambda_{12}-\eta\right) \sin \left(\lambda_{1}+\bar{\xi}+u+\eta\right)}{\sin \lambda_{12} \sin \left(\lambda_{1}+\bar{\xi}-u-\eta\right)}, \frac{\sin \left(\lambda_{12}+\eta\right) \sin \left(\lambda_{2}+\bar{\xi}+u+\eta\right)}{\sin \lambda_{12} \sin \left(\lambda_{2}+\bar{\xi}-u-\eta\right)}\right) .
\end{aligned}
$$

Although the vertex type K-matrices $K^{ \pm}(u)$ given by (2.8) and (2.10) are generally nondiagonal, after the face-vertex transformations (2.35) and (2.36), the face type counterparts $\mathcal{K}(\lambda \mid u)$ and $\tilde{\mathcal{K}}(\lambda \mid u)$ become simultaneously diagonal. This fact enabled the authors to apply the generalized algebraic Bethe ansatz method developed in [16] for SOS type integrable models to diagonalize the transfer matrices $\tau(u)(2.7)$ [20, 28]. 


\section{$2.2 \quad$ Two sets of eigenstates}

By (2.36) and (2.38), we can recast the transfer matrix $\tau(u)(2.7)$ in the form

$$
\tau(u)=\operatorname{tr}\left(K^{+}(u) \mathbb{T}(u)\right)=\sum_{\mu, \nu} \tilde{\mathcal{K}}(\lambda \mid u)_{\nu}^{\mu} \mathcal{T}^{-}(\lambda \mid u)_{\mu}^{\nu}=\sum_{\mu} \tilde{k}(\lambda \mid u)_{\mu} \mathcal{T}^{-}(\lambda \mid u)_{\mu}^{\mu}
$$

Here we have introduced the new double-row monodromy matrix $\mathcal{T}^{-}(m \mid u)$

$$
\mathcal{T}^{-}(m \mid u)_{\mu}^{\nu}=\tilde{\phi}_{m-\eta(\hat{\mu}-\hat{\nu}), m-\eta \hat{\mu}}^{0}(u) \mathbb{T}_{0}(u) \phi_{m, m-\eta \hat{\mu}}^{0}(-u)
$$

This double-row monodromy matrix, in the face picture, can be expressed in terms of the face type R-matrix $R(u ; m)$ (2.16) and K-matrix $\mathcal{K}(\lambda \mid u)$ (2.37) (for the details, see (4.22) below).

In contrast with the case of diagonal boundary terms [13], there exist two sets of Bethe states which constitute the complete set of eigenstates of the transfer matrix for the models with non-diagonal boundary terms [28]. These two sets of states are given by

$$
\begin{aligned}
& \left|v_{1}^{(1)}, \cdots, v_{M}^{(1)}\right\rangle^{(1)}=\mathcal{T}^{-}\left(\lambda-2 \eta \hat{1} \mid v_{1}^{(1)}\right)_{2}^{1} \cdots \mathcal{T}^{-}\left(\lambda-2 M \eta \hat{1} \mid v_{M}^{(1)}\right)_{2}^{1}\left|\Omega^{(1)}(\lambda)\right\rangle, \\
& \left|v_{1}^{(2)}, \cdots, v_{M}^{(2)}\right\rangle^{(I I)}=\mathcal{T}^{-}\left(\lambda-2 \eta \hat{2} \mid v_{1}^{(2)}\right)_{1}^{2} \cdots \mathcal{T}^{-}\left(\lambda-2 M \eta \hat{2} \mid v_{M}^{(2)}\right)_{1}^{2}\left|\Omega^{(I I)}(\lambda)\right\rangle,
\end{aligned}
$$

where the vector $\lambda$ is related to the boundary parameters (2.12). The associated reference states $\left|\Omega^{(1)}(\lambda)\right\rangle$ and $\left|\Omega^{(I I)}(\lambda)\right\rangle$ are

$$
\begin{aligned}
\left|\Omega^{(1)}(\lambda)\right\rangle & =\phi_{\lambda, \lambda-\eta \hat{1}}^{1}\left(z_{1}\right) \phi_{\lambda-\eta \hat{1}, \lambda-2 \eta \hat{1}}^{2}\left(z_{2}\right) \cdots \phi_{\lambda-(N-1) \eta \hat{1}, \lambda-N \eta \hat{1}}^{N}\left(z_{N}\right), \\
\left|\Omega^{(I I)}(\lambda)\right\rangle & =\phi_{\lambda, \lambda-\eta \hat{2}}^{1}\left(z_{1}\right) \phi_{\lambda-\eta \hat{2}, \lambda-2 \eta \hat{2}}^{2}\left(z_{2}\right) \cdots \phi_{\lambda-(N-1) \eta \hat{2}, \lambda-N \eta \hat{2}}^{N}\left(z_{N}\right) .
\end{aligned}
$$

It is remarked that $\phi^{k}=\mathrm{id} \otimes \mathrm{id} \cdots \otimes \stackrel{k-t h}{\phi} \otimes \mathrm{id} \cdots$.

If the parameters $\left\{v_{k}^{(1)}\right\}$ satisfy the first set of Bethe ansatz equations,

$$
\begin{aligned}
\frac{\sin \left(\lambda_{2}+\xi+v_{\alpha}^{(1)}\right) \sin \left(\lambda_{2}+\bar{\xi}-v_{\alpha}^{(1)}\right) \sin \left(\lambda_{1}+\bar{\xi}+v_{\alpha}^{(1)}\right) \sin \left(\lambda_{1}+\xi-v_{\alpha}^{(1)}\right)}{\sin \left(\lambda_{2}+\bar{\xi}+v_{\alpha}^{(1)}+\eta\right) \sin \left(\lambda_{2}+\xi-v_{\alpha}^{(1)}-\eta\right) \sin \left(\lambda_{1}+\xi+v_{\alpha}^{(1)}+\eta\right) \sin \left(\lambda_{1}+\bar{\xi}-v_{\alpha}^{(1)}-\eta\right)} \\
=\prod_{k \neq \alpha}^{M} \frac{\sin \left(v_{\alpha}^{(1)}+v_{k}^{(1)}+2 \eta\right) \sin \left(v_{\alpha}^{(1)}-v_{k}^{(1)}+\eta\right)}{\sin \left(v_{\alpha}^{(1)}+v_{k}^{(1)}\right) \sin \left(v_{\alpha}^{(1)}-v_{k}^{(1)}-\eta\right)} \\
\quad \times \prod_{k=1}^{2 M} \frac{\sin \left(v_{\alpha}^{(1)}+z_{k}\right) \sin \left(v_{\alpha}^{(1)}-z_{k}\right)}{\sin \left(v_{\alpha}^{(1)}+z_{k}+\eta\right) \sin \left(v_{\alpha}^{(1)}-z_{k}+\eta\right)}, \quad \alpha=1, \cdots, M,
\end{aligned}
$$


the Bethe state $\left|v_{1}^{(1)}, \cdots, v_{M}^{(1)}\right\rangle^{(1)}$ becomes the eigenstate of the transfer matrix with eigenvalue $\Lambda^{(1)}(u)$ given by $[20$ ]

$$
\begin{aligned}
\Lambda^{(1)}(u)=\frac{\sin \left(\lambda_{2}+\bar{\xi}-u\right) \sin \left(\lambda_{1}+\bar{\xi}+u\right) \sin \left(\lambda_{1}+\xi-u\right) \sin (2 u+2 \eta)}{\sin \left(\lambda_{2}+\bar{\xi}-u-\eta\right) \sin \left(\lambda_{1}+\bar{\xi}-u-\eta\right) \sin \left(\lambda_{1}+\xi+u\right) \sin (2 u+\eta)} \\
\quad \times \prod_{k=1}^{M} \frac{\sin \left(u+v_{k}^{(1)}\right) \sin \left(u-v_{k}^{(1)}-\eta\right)}{\sin \left(u+v_{k}^{(1)}+\eta\right) \sin \left(u-v_{k}^{(1)}\right)} \\
+\frac{\sin \left(\lambda_{2}+\bar{\xi}+u+\eta\right) \sin \left(\lambda_{1}+\xi+u+\eta\right) \sin \left(\lambda_{2}+\xi-u-\eta\right) \sin 2 u}{\sin \left(\lambda_{2}+\bar{\xi}-u-\eta\right) \sin \left(\lambda_{1}+\xi+u\right) \sin \left(\lambda_{2}+\xi+u\right) \sin (2 u+\eta)} \\
\quad \times \prod_{k=1}^{M} \frac{\sin \left(u+v_{k}^{(1)}+2 \eta\right) \sin \left(u-v_{k}^{(1)}+\eta\right)}{\sin \left(u+v_{k}^{(1)}+\eta\right) \sin \left(u-v_{k}^{(1)}\right)} \\
\quad \times \prod_{k=1}^{2 M} \frac{\sin \left(u+z_{k}\right) \sin \left(u-z_{k}\right)}{\sin \left(u+z_{k}+\eta\right) \sin \left(u-z_{k}+\eta\right)}
\end{aligned}
$$

If the parameters $\left\{v_{k}^{(2)}\right\}$ satisfy the second Bethe Ansatz equations

$$
\begin{aligned}
\frac{\sin \left(\lambda_{1}+\xi+v_{\alpha}^{(2)}\right) \sin \left(\lambda_{1}+\bar{\xi}-v_{\alpha}^{(2)}\right) \sin \left(\lambda_{2}+\bar{\xi}+v_{\alpha}^{(2)}\right) \sin \left(\lambda_{2}+\xi-v_{\alpha}^{(2)}\right)}{\sin \left(\lambda_{1}+\bar{\xi}+v_{\alpha}^{(2)}+\eta\right) \sin \left(\lambda_{1}+\xi-v_{\alpha}^{(2)}-\eta\right) \sin \left(\lambda_{2}+\xi+v_{\alpha}^{(2)}+\eta\right) \sin \left(\lambda_{2}+\bar{\xi}-v_{\alpha}^{(2)}-\eta\right)} \\
=\prod_{k \neq \alpha}^{M} \frac{\sin \left(v_{\alpha}^{(2)}+v_{k}^{(2)}+2 \eta\right) \sin \left(v_{\alpha}^{(2)}-v_{k}^{(2)}+\eta\right)}{\sin \left(v_{\alpha}^{(2)}+v_{k}^{(2)}\right) \sin \left(v_{\alpha}^{(2)}-v_{k}^{(2)}-\eta\right)} \\
\quad \times \prod_{k=1}^{2 M} \frac{\sin \left(v_{\alpha}^{(2)}+z_{k}\right) \sin \left(v_{\alpha}^{(2)}-z_{k}\right)}{\sin \left(v_{\alpha}^{(2)}+z_{k}+\eta\right) \sin \left(v_{\alpha}^{(2)}-z_{k}+\eta\right)}, \quad \alpha=1, \cdots, M,
\end{aligned}
$$

the Bethe states $\left|v_{1}^{(2)}, \cdots, v_{M}^{(2)}\right\rangle^{(I I)}$ yield the second set of the eigenstates of the transfer matrix with the eigenvalues [28],

$$
\begin{aligned}
\Lambda^{(2)}(u)=\frac{\sin (2 u+2 \eta) \sin \left(\lambda_{1}+\bar{\xi}-u\right) \sin \left(\lambda_{2}+\bar{\xi}+u\right) \sin \left(\lambda_{2}+\xi-u\right)}{\sin (2 u+\eta) \sin \left(\lambda_{1}+\bar{\xi}-u-\eta\right) \sin \left(\lambda_{2}+\bar{\xi}-u-\eta\right) \sin \left(\lambda_{2}+\xi+u\right)} \\
\quad \times \prod_{k=1}^{M} \frac{\sin \left(u+v_{k}^{(2)}\right) \sin \left(u-v_{k}^{(2)}-\eta\right)}{\sin \left(u+v_{k}^{(2)}+\eta\right) \sin \left(u-v_{k}^{(2)}\right)} \\
+\frac{\sin (2 u)}{\sin \left(\lambda_{1}+\bar{\xi}+u+\eta\right) \sin \left(\lambda_{2}+\xi+u+\eta\right) \sin \left(\lambda_{1}+\xi-u-\eta\right)} \\
\quad \times \prod_{k=1}^{M} \frac{\sin \left(u+v_{k}^{(2)}+2 \eta\right) \sin \left(u-v_{k}^{(2)}+\eta\right)}{\sin \left(u+v_{k}^{(2)}+\eta\right) \sin \left(u-v_{k}^{(2)}\right)} \\
\times \prod_{k=1}^{2 M} \frac{\sin \left(u+z_{k}\right) \sin \left(u-z_{k}\right)}{\sin \left(u+z_{k}+\eta\right) \sin \left(u-z_{k}+\eta\right)} .
\end{aligned}
$$




\section{A new set of Bethe states}

In contrast with the case of periodic boundary conditions [2], we find that in the F-basis $\mathcal{T}^{-}(m \mid u)_{2}^{1}$ and $\mathcal{T}^{-}(m \mid u)_{1}^{2}$ given in (2.40) cannot simultaneously be expressed in simple polarization free form. A similar situation already appeared in the open XXZ chain with diagonal K-matrices [11, 12]. However, such polarization free forms of the pseudo-particle creation operators are crucial for computing partition functions and scalar products [4, 10, 9, 12, 34].

To overcome the difficulty, let us introduce another double-row monodromy matrix $\mathcal{T}^{+}(m \mid u)$ (c.f. $\mathcal{T}^{-}(m \mid u)$ given by (2.40) $)$

$$
\begin{aligned}
\mathcal{T}^{+}(m \mid u)_{i}^{j} & =\prod_{k \neq j} \frac{\sin \left(m_{j k}\right)}{\sin \left(m_{j k}-\eta\right)} \phi_{m-\eta(\hat{\jmath}-\hat{\imath}), m-\eta \hat{\jmath}}^{t_{0}}(u)\left(\mathbb{T}^{+}(u)\right)^{t_{0}} \bar{\phi}_{m, m-\eta \hat{\jmath}}^{t_{0}}(-u), \\
\left(\mathbb{T}^{+}(u)\right)^{t_{0}} & =T^{t_{0}}(u)\left(K^{+}(u)\right)^{t_{0}} \hat{T}^{t_{0}}(u),
\end{aligned}
$$

where $t_{0}$ denotes transposition in the 0 -th space (i.e. auxiliary space). As will be shown in section 5 , in the F-basis the face versions of the operators $\mathcal{T}^{+}(m \mid u)_{2}^{1}$ and $\mathcal{T}^{-}(m \mid u)_{1}^{2}$ become completely symmetric and polarization free simultaneously (see (5.20) and (5.21) below). Thus $\mathcal{T}^{+}(m \mid u)_{2}^{1}$ is the desirable pseudo-particle creation operator which generates the new set of Bethe states $\left|v_{1}^{(1)}, \cdots, v_{M}^{(1)}\right\rangle^{(I)}$, in replacement of the first set of Bethe states (2.41),

$$
\left|v_{1}^{(1)}, \cdots, v_{M}^{(1)}\right\rangle^{(I)}=\mathcal{T}^{+}\left(\lambda+2 \eta \hat{1} \mid v_{1}^{(1)}\right)_{2}^{1} \cdots \mathcal{T}^{+}\left(\lambda+2 M \eta \hat{1} \mid v_{M}^{(1)}\right)_{2}^{1}\left|\Omega^{(I)}(\lambda)\right\rangle
$$

with reference state $\left|\Omega^{(I)}(\lambda)\right\rangle$ (c.f. $\left|\Omega^{(1)}(\lambda)\right\rangle$ given by (2.43) $)$

$$
\left|\Omega^{(I)}(\lambda)\right\rangle=\phi_{\lambda+N \eta \hat{1}, \lambda+(N-1) \eta \hat{1}}^{1}\left(z_{1}\right) \phi_{\lambda+(N-1) \eta \hat{1}, \lambda+(N-2) \eta \hat{1}}^{2}\left(z_{2}\right) \cdots \phi_{\lambda+\eta \hat{1}, \lambda}^{N}\left(z_{N}\right) .
$$

As will be seen in section 5, the two sets of Bethe states (3.3) and (2.42) are of completely symmetric form in the F-basis. Moreover, using the technique developed in [16], we find that if the parameters $\left\{v_{k}^{(1)}\right\}$ in (3.3) satisfy the first set of Bethe ansatz equations (2.45),

the state $\left|v_{1}^{(1)}, \cdots, v_{M}^{(1)}\right\rangle^{(I)}$ is the eigenstate of the transfer matrix with eigenvalue $\Lambda^{(1)}(u)$ given by (2.46) (for details, see Appendix A). Hence two sets of Bethe states (3.3) and (2.42) constitute a complete set.

\section{$4 \mathcal{T}^{ \pm}(m \mid u)$ in the face picture}

The K-matrices $K^{ \pm}(u)$ given by (2.8) and (2.10) are generally non-diagonal (in the vertex picture), after the face-vertex transformations (2.35) and (2.36), the face type counterparts 
$\mathcal{K}(\lambda \mid u)$ and $\tilde{\mathcal{K}}(\lambda \mid u)$ given by (2.37) and (2.38) simultaneously become diagonal. This fact suggests that it would be much simpler if one performs all calculations in the face picture.

Let us introduce the face type one-row monodromy matrix (c.f (2.3))

$$
\begin{aligned}
T_{F}(l \mid u) & \equiv T_{0,1 \ldots N}^{F}(l \mid u) \\
& =R_{0, N}\left(u-z_{N} ; l-\eta \sum_{i=1}^{N-1} h^{(i)}\right) \ldots R_{0,2}\left(u-z_{2} ; l-\eta h^{(1)}\right) R_{0,1}\left(u-z_{1} ; l\right), \\
& =\left(\begin{array}{cc}
T_{F}(l \mid u)_{1}^{1} & T_{F}(l \mid u)_{2}^{1} \\
T_{F}(l \mid u)_{1}^{2} & T_{F}(l \mid u)_{2}^{2}
\end{array}\right)
\end{aligned}
$$

where $l$ is a generic vector in $V$. The monodromy matrix satisfies the face type quadratic exchange relation [35, 36]. Applying $T_{F}(l \mid u)_{j}^{i}$ to an arbitrary vector $\left|i_{1}, \ldots, i_{N}\right\rangle$ in the Ntensor product space $V^{\otimes N}$ given by

$$
\left|i_{1}, \ldots, i_{N}\right\rangle=\epsilon_{i_{1}}^{1} \ldots \epsilon_{i_{N}}^{N}
$$

we have

$$
\begin{aligned}
& T_{F}(l \mid u)_{j}^{i}\left|i_{1}, \ldots, i_{N}\right\rangle \equiv T_{F}(m ; l \mid u)_{j}^{i}\left|i_{1}, \ldots, i_{N}\right\rangle \\
&= \sum_{\alpha_{N-1} \ldots \alpha_{1} i_{N}^{\prime} \ldots i_{1}^{\prime}} R\left(u-z_{N} ; l-\eta \sum_{k=1}^{N-1} \hat{\imath}_{k}^{\prime}\right)_{\alpha_{N-1} i_{N}}^{i} \ldots \\
& \quad \quad \times R\left(u-z_{2} ; l-\eta \hat{\imath}_{1}^{\prime}\right)_{\alpha_{1} i_{2}}^{\alpha_{2} i_{2}^{\prime}} R\left(u-z_{1} ; l\right)_{j}^{\alpha_{1} i_{1}^{\prime}}\left|i_{1}^{\prime}, \ldots, i_{N}^{\prime}\right\rangle,
\end{aligned}
$$

where $m=l-\eta \sum_{k=1}^{N} \hat{\imath}_{k}$. We shall express the double-row monodromy matrices $\mathcal{T}^{ \pm}$given by (2.40) and (3.1) in terms of the above face-type one-row monodromy matrix.

Associated with the vertex type monodromy matrices $T(u)(2.3)$ and $\hat{T}(u)$ (2.6), we introduce the following operators

$$
\begin{aligned}
T(m, l \mid u)_{\mu}^{j} & =\tilde{\phi}_{m+\eta \hat{\jmath}, m}^{0}(u) T_{0}(u) \phi_{l+\eta \hat{\mu}, l}^{0}(u), \\
S(m, l \mid u)_{i}^{\mu} & =\bar{\phi}_{l, l-\eta \hat{\mu}}^{0}(-u) \hat{T}_{0}(u) \phi_{m, m-\eta \hat{\imath}}^{0}(-u) .
\end{aligned}
$$

Moreover, for the case of $m=l-\eta \sum_{k=1}^{N} \hat{\imath}_{k}$, we introduce a generic state in the quantum space from the intertwiner vector (2.25)

$$
\left|i_{1}, \ldots, i_{N}\right\rangle_{l}^{m}=\phi_{l, l-\eta \hat{\imath}_{1}}^{1}\left(z_{1}\right) \phi_{l-\eta \hat{\imath}_{1}, l-\eta\left(\hat{\imath}_{1}+\hat{\imath}_{2}\right)}^{2}\left(z_{2}\right) \ldots \phi_{l-\eta \sum_{k=1}^{N-1} \hat{\imath}_{k}, l-\eta \sum_{k=1}^{N} \hat{\imath}_{k}}^{N}\left(z_{N}\right) .
$$


We can evaluate the action of the operator $T(m, l \mid u)$ on the state $\left|i_{1}, \ldots, i_{N}\right\rangle_{l}^{m}$ from the face-vertex correspondence relation (2.27)

$$
\begin{aligned}
& T(m, l \mid u)_{\mu}^{j}\left|i_{1}, \ldots, i_{N}\right\rangle_{l}^{m}=\tilde{\phi}_{m+\eta \hat{\jmath}, m}^{0}(u) T_{0}(u) \phi_{l+\eta \hat{\mu}, l}^{0}(u)\left|i_{1}, \ldots, i_{N}\right\rangle_{l}^{m} \\
& =\tilde{\phi}_{m+\eta \hat{\jmath}, m}^{0}(u) \bar{R}_{0, N}\left(u-z_{N}\right) \ldots \bar{R}_{0,1}\left(u-z_{1}\right) \phi_{l+\eta \hat{\mu}, l}^{0}(u) \phi_{l, l-\eta \hat{\imath}_{1}}^{1}\left(z_{1}\right) \ldots \\
& =\sum_{\alpha_{1}, i_{1}^{\prime}} R\left(u-z_{1} ; l+\eta \hat{\mu}\right)_{\mu i_{1}}^{\alpha_{1} i_{1}^{\prime}} \phi_{l+\eta \hat{\mu}, l+\eta \hat{\mu}-\eta \hat{\imath}_{1}^{\prime}}^{1}\left(z_{1}\right) \tilde{\phi}_{m+\eta \hat{\jmath}, m}^{0}(u) \bar{R}_{0, N}\left(u-z_{N}\right) \ldots \\
& \quad \quad \times \bar{R}_{0,2}\left(u-z_{2}\right) \phi_{l+\eta \hat{\mu}-\eta \hat{\imath}_{1}^{\prime}, l-\eta \hat{\imath}_{1}}^{0}(u) \phi_{l-\eta \hat{\imath}_{1}, l-\eta\left(\hat{\imath}_{1}+\hat{\imath}_{2}\right)}^{2}\left(z_{2}\right) \ldots \\
& \quad \vdots \quad \sum_{\quad} \quad \sum_{\alpha_{1} \ldots \alpha_{N-1}} \sum_{i_{1}^{\prime} \ldots i_{N}^{\prime}} R\left(u-z_{N} ; l+\eta \hat{\mu}-\eta \sum_{k=1}^{N-1} \hat{\imath}_{k}^{\prime}\right)_{\alpha_{N-1} i_{N} i_{N}^{\prime}}^{i_{1}^{\prime}} \ldots \\
& \quad \times R\left(u-z_{1} ; l+\eta \hat{\mu}\right)_{\mu i_{1}}^{\alpha_{1} i_{1}^{\prime}}\left|i_{1}^{\prime}, \ldots, i_{N}^{\prime}\right\rangle_{l+\eta \hat{\mu}}^{l+\eta \hat{\mu}-\eta \sum_{k=1}^{N} \hat{\imath}_{k}^{\prime}} .
\end{aligned}
$$

Here we have used the following property of the R-matrix

$$
R(u ; m)_{i j}^{i^{\prime} j^{\prime}}=R(u ; m \pm \eta(\hat{\imath}+\hat{\jmath}))_{i j}^{i^{i^{\prime} j^{\prime}}}=R\left(u ; m \pm \eta\left(\hat{\imath}^{\prime}+\hat{\jmath}^{\prime}\right)\right)_{i j}^{i^{\prime} j^{\prime}}
$$

and the weight conservation condition (2.21). Comparing with (4.3), we have the following correspondence

$$
T(m, l \mid u)_{\mu}^{j}\left|i_{1}, \ldots, i_{N}\right\rangle_{l}^{m} \longleftrightarrow T_{F}(m+\eta \hat{\mu} ; l+\eta \hat{\mu} \mid u)_{\mu}^{j}\left|i_{1}, \ldots, i_{N}\right\rangle
$$

where vector $\left|i_{1}, \ldots, i_{N}\right\rangle$ is given by (4.2). Hereafter, we will use $O_{F}$ to denote the face version of operator $O$ in the face picture.

Noting that

$$
\hat{T}_{0}(u)=\bar{R}_{1,0}\left(u+z_{1}\right) \ldots \bar{R}_{N, 0}\left(u+z_{N}\right)
$$

we obtain the action of $S(m, l \mid u)_{i}^{\mu}$ on the state $\left|i_{1}, \ldots, i_{N}\right\rangle_{l}^{m}$

$$
\begin{aligned}
S(m, l \mid u)_{i}^{\mu}\left|i_{1}, \ldots, i_{N}\right\rangle_{l}^{m}= & \sum_{\alpha_{1} \ldots \alpha_{N-1}} \sum_{i_{1}^{\prime} \ldots i_{N}^{\prime}} R\left(u+z_{1} ; l\right)_{i_{1} \alpha_{N-1}}^{i_{1}^{\prime} \mu} R\left(u+z_{2} ; l-\eta \hat{\imath}_{1}\right)_{i_{2} \alpha_{N-2}}^{i_{2}^{\prime} \alpha_{N-1}} \\
& \times \ldots R\left(u+z_{N} ; l-\eta \sum_{k=1}^{N-1} \hat{\imath}_{k}\right)_{i_{N} i}^{i_{N}^{\prime} \alpha_{1}}\left|i_{1}^{\prime}, \ldots, i_{N}^{\prime}\right\rangle_{l-\eta \hat{\mu}}^{l-\eta \hat{\mu}-\eta \sum_{k=1}^{N} \hat{\imath}_{k}^{\prime}} .
\end{aligned}
$$


Then the crossing relation of the R-matrix (2.23) enables us to establish the following relation:

$$
S(m, l \mid u)_{i}^{\mu}=\varepsilon_{\bar{i}} \varepsilon_{\bar{\mu}} \frac{\sin \left(m_{21}\right)}{\sin \left(l_{21}\right)} \prod_{k=1}^{N} \frac{\sin \left(u+z_{k}\right)}{\sin \left(u+z_{k}+\eta\right)} T(m, l \mid-u-\eta)_{\bar{\mu}}^{\bar{i}},
$$

where the parities are defined in (2.24) and $m_{21}$ (or $\left.l_{21}\right)$ is defined in (2.15) .

Now we are in the position to express $\mathcal{T}^{ \pm}(2.40)$ and (3.1) in terms of $T(m, l)_{j}^{i}$ and $S(l, m)_{j}^{i}$. By (2.29) and (2.30), we have

$$
\begin{aligned}
\mathcal{T}^{-}(m \mid u)_{i}^{j}= & \tilde{\phi}_{m-\eta(\hat{\imath}-\hat{\jmath}), m-\eta \hat{\imath}}^{0}(u) \mathbb{T}(u) \phi_{m, m-\eta \hat{\imath}}^{0}(-u) \\
= & \tilde{\phi}_{m-\eta(\hat{\imath}-\hat{\jmath}), m-\eta \hat{\imath}}^{0}(u) T_{0}(u) K_{0}^{-}(u) \hat{T}_{0}(u) \phi_{m, m-\eta \hat{\imath}}^{0}(-u) \\
= & \sum_{\mu, \nu} \tilde{\phi}_{m-\eta(\hat{\imath}-\hat{\jmath}), m-\eta \hat{\imath}}^{0}(u) T_{0}(u) \phi_{l-\eta(\hat{\nu}-\hat{\mu}), l-\eta \hat{\nu}}^{0}(u) \tilde{\phi}_{l-\eta(\hat{\nu}-\hat{\mu}), l-\eta \hat{\nu}}^{0}(u) \\
& \times K_{0}^{-}(u) \phi_{l, l-\eta \hat{\nu}}^{0}(-u) \bar{\phi}_{l, l-\eta \hat{\nu}}^{0}(-u) \hat{T}_{0}(u) \phi_{m, m-\eta \hat{\imath}}^{0}(-u) \\
= & \sum_{\mu, \nu} T(m-\eta \hat{\imath}, l-\eta \hat{\nu} \mid u)_{\mu}^{j} \mathcal{K}(l \mid u)_{\nu}^{\mu} S(m, l \mid u)_{i}^{\nu} \\
& \stackrel{\text { def }}{=} \mathcal{T}^{-}(m, l \mid u)_{i}^{j},
\end{aligned}
$$

where the face-type K-matrix $\mathcal{K}(l \mid u)_{\nu}^{\mu}$ is given by

$$
\mathcal{K}(l \mid u)_{\nu}^{\mu}=\tilde{\phi}_{l-\eta(\hat{\nu}-\hat{\mu}), l-\eta \hat{\nu}}^{0}(u) K_{0}^{-}(u) \phi_{l, l-\eta \hat{\nu}}^{0}(-u)
$$

Similarly, we have

$$
\begin{aligned}
\mathcal{T}^{+}(m \mid u)_{i}^{j} & =\prod_{k \neq j} \frac{\sin m_{j k}}{\sin \left(m_{j k}-\eta\right)} \sum_{\mu, \nu} T(l-\eta \hat{\mu}, m-\eta \hat{\jmath} \mid u)_{i}^{\nu} \tilde{\mathcal{K}}(l \mid u)_{\nu}^{\mu} S(l, m \mid u)_{\mu}^{j} \\
& \stackrel{\text { def }}{=} \mathcal{T}^{+}(l, m \mid u)_{i}^{j}
\end{aligned}
$$

with

$$
\tilde{\mathcal{K}}(l \mid u)_{\nu}^{\mu}=\bar{\phi}_{l, l-\eta \hat{\mu}}^{0}(-u) K_{0}^{+}(u) \phi_{l-\eta(\hat{\mu}-\hat{\nu}), l-\eta \hat{\mu}}^{0}(u) .
$$

Thanks to the fact that when $l=\lambda$ the corresponding face-type K-matrices $\mathcal{K}(\lambda \mid u)(4.13)$ and $\tilde{\mathcal{K}}(\lambda \mid u)$ (4.15) become diagonal ones (2.37) and (2.38), we have

$$
\begin{aligned}
\mathcal{T}^{-}(m, \lambda \mid u)_{i}^{j} & =\sum_{\mu} T(m-\eta \hat{\imath}, \lambda-\eta \hat{\mu} \mid u)_{\mu}^{j} k(\lambda \mid u)_{\mu} S(m, \lambda \mid u)_{i}^{\mu}, \\
\mathcal{T}^{+}(\lambda, m \mid u)_{i}^{j} & =\prod_{k \neq j} \frac{\sin m_{j k}}{\sin \left(m_{j k}-\eta\right)} \sum_{\mu} T(\lambda-\eta \hat{\mu}, m-\eta \hat{\jmath} \mid u)_{i}^{\mu} \tilde{k}(\lambda \mid u)_{\mu} S(\lambda, m \mid u)_{\mu}^{j},
\end{aligned}
$$


where the functions $k(\lambda \mid u)_{\mu}$ and $\tilde{k}(\lambda \mid u)_{\mu}$ are given by (2.37) and (2.38) respectively. The relation (4.11) implies that one can further express $\mathcal{T}^{ \pm}(m \mid u)_{i}^{j}$ in terms of only $T(m, l \mid u)_{i}^{j}$. Here we present the results for the pseudo-particle creation operators $\mathcal{T}^{-}(m \mid u)_{1}^{2}$ in (2.42) and $\mathcal{T}^{+}(m \mid u)_{2}^{1}$ in $(3.3)$ :

$$
\begin{aligned}
\mathcal{T}^{-}(m \mid u)_{1}^{2}=\mathcal{T}^{-} & (m, \lambda \mid u)_{1}^{2}=\frac{\sin \left(m_{21}\right)}{\sin \left(\lambda_{21}\right)} \prod_{k=1}^{N} \frac{\sin \left(u+z_{k}\right)}{\sin \left(u+z_{k}+\eta\right)} \\
& \times\left\{\frac{\sin \left(\lambda_{1}+\xi-u\right)}{\sin \left(\lambda_{1}+\xi+u\right)} T(m+\eta \hat{2}, \lambda+\eta \hat{2} \mid u)_{1}^{2} T(m, \lambda \mid-u-\eta)_{2}^{2}\right. \\
& \left.\quad-\frac{\sin \left(\lambda_{2}+\xi-u\right)}{\sin \left(\lambda_{2}+\xi+u\right)} T(m+\eta \hat{2}, \lambda+\eta \hat{1} \mid u)_{2}^{2} T(m, \lambda \mid-u-\eta)_{1}^{2}\right\}, \\
\mathcal{T}^{+}(m \mid u)_{2}^{1}=\mathcal{T}^{+}(\lambda, m \mid u)_{2}^{1}=\prod_{k=1}^{N} \frac{\sin \left(u+z_{k}\right)}{\sin \left(u+z_{k}+\eta\right)} & \times\left\{\frac{\sin \left(\lambda_{12}-\eta\right) \sin \left(\lambda_{1}+\bar{\xi}+u+\eta\right)}{\sin \left(m_{12}-\eta\right) \sin \left(\lambda_{1}+\bar{\xi}-u-\eta\right)} T(\lambda+\eta \hat{2}, m+\eta \hat{2} \mid u)_{2}^{1} T(\lambda, m \mid-u-\eta)_{2}^{2}\right. \\
& \left.\quad-\frac{\sin \left(\lambda_{21}-\eta\right) \sin \left(\lambda_{2}+\bar{\xi}+u+\eta\right)}{\sin \left(m_{21}+\eta\right) \sin \left(\lambda_{2}+\bar{\xi}-u-\eta\right)} T(\lambda+\eta \hat{1}, m+\eta \hat{2} \mid u)_{2}^{2} T(\lambda, m \mid-u-\eta)_{2}^{1}\right\} .
\end{aligned}
$$

Similar to (4.9), we have the correspondence,

$$
\begin{aligned}
\mathcal{T}^{-}(m, l \mid u)_{1}^{2}\left|i_{1}, \ldots, i_{N}\right\rangle_{l}^{m} & \mathcal{T}_{F}^{-}(m, l \mid u)_{1}^{2}\left|i_{1}, \ldots, i_{N}\right\rangle \\
\mathcal{T}^{+}(m, l \mid u)_{2}^{1}\left|i_{1}, \ldots, i_{N}\right\rangle_{l}^{m} & \longleftrightarrow \mathcal{T}_{F}^{+}(m, l \mid u)_{2}^{1}\left|i_{1}, \ldots, i_{N}\right\rangle
\end{aligned}
$$

It follows from (4.18) and (4.19) that the face-type double-row monodromy matrix elements $\mathcal{T}_{F}^{-}(m, \lambda \mid u)_{1}^{2}$ and $\mathcal{T}_{F}^{+}(\lambda, m \mid u)_{2}^{1}$ can be expressed in terms of the face-type one-row monodromy matrix elements $T_{F}(m, l \mid u)_{j}^{i}(4.3)$ by

$$
\begin{aligned}
& \mathcal{T}_{F}^{-}(m, \lambda \mid u)_{1}^{2}=\frac{\sin \left(m_{21}\right)}{\sin \left(\lambda_{21}\right)} \prod_{k=1}^{N} \frac{\sin \left(u+z_{k}\right)}{\sin \left(u+z_{k}+\eta\right)} \\
& \quad \times\left\{\frac{\sin \left(\lambda_{1}+\xi-u\right)}{\sin \left(\lambda_{1}+\xi+u\right)} T_{F}(m, \lambda \mid u)_{1}^{2} T_{F}(m+\eta \hat{2}, \lambda+\eta \hat{2} \mid-u-\eta)_{2}^{2}\right. \\
& \left.\quad-\frac{\sin \left(\lambda_{2}+\xi-u\right)}{\sin \left(\lambda_{2}+\xi+u\right)} T_{F}(m+2 \eta \hat{2}, \lambda \mid u)_{2}^{2} T_{F}(m+\eta \hat{1}, \lambda+\eta \hat{1} \mid-u-\eta)_{1}^{2}\right\} \\
& \mathcal{T}_{F}^{+}(\lambda, m \mid u)_{2}^{1}=\prod_{k=1}^{N} \frac{\sin \left(u+z_{k}\right)}{\sin \left(u+z_{k}+\eta\right)} \\
& \quad \times\left\{\frac{\sin \left(\lambda_{12}-\eta\right) \sin \left(\lambda_{1}+\bar{\xi}+u+\eta\right)}{\sin \left(m_{12}-\eta\right) \sin \left(\lambda_{1}+\bar{\xi}-u-\eta\right)} T_{F}(\lambda+2 \eta \hat{2}, m+2 \eta \hat{2} \mid u)_{2}^{1} T_{F}(\lambda+\eta \hat{2}, m+\eta \hat{2} \mid-u-\eta)_{2}^{2}\right.
\end{aligned}
$$




$$
\left.-\frac{\sin \left(\lambda_{21}-\eta\right) \sin \left(\lambda_{2}+\bar{\xi}+u+\eta\right)}{\sin \left(m_{21}+\eta\right) \sin \left(\lambda_{2}+\bar{\xi}-u-\eta\right)} T_{F}(\lambda, m+2 \eta \hat{2} \mid u)_{2}^{2} T_{F}(\lambda+\eta \hat{2}, m+\eta \hat{2} \mid-u-\eta)_{2}^{1}\right\}
$$

In the derivation of the above equations we have used the identity $\hat{1}+\hat{2}=0$. Finally, we obtain the face versions $\left|v_{1}^{(1)}, \cdots, v_{M}^{(1)}\right\rangle_{F}^{(I)}$ and $\left|v_{1}^{(1)}, \cdots, v_{M}^{(1)}\right\rangle_{F}^{(I I)}$ of the two sets of Bethe states (3.3) and (2.42),

$$
\begin{aligned}
& \left|v_{1}^{(1)}, \cdots, v_{M}^{(1)}\right\rangle_{F}^{(I)}=\mathcal{T}_{F}^{+}\left(\lambda, \lambda+2 \eta \hat{1} \mid v_{1}^{(1)}\right)_{2}^{1} \cdots \mathcal{T}_{F}^{+}\left(\lambda, \lambda+2 M \eta \hat{1} \mid v_{M}^{(1)}\right)_{2}^{1}|1, \ldots, 1\rangle \\
& \left|v_{1}^{(2)}, \cdots, v_{M}^{(2)}\right\rangle_{F}^{(I I)}=\mathcal{T}_{F}^{-}\left(\lambda-2 \eta \hat{2}, \lambda \mid v_{1}^{(2)}\right)_{1}^{2} \cdots \mathcal{T}_{F}^{-}\left(\lambda-2 M \eta \hat{2}, \lambda \mid v_{M}^{(2)}\right)_{1}^{2}|2, \ldots, 2\rangle
\end{aligned}
$$

In the next section we shall construct the Drinfeld twist (or factorizing F-matrix) in the face picture for the open XXZ chain with non-diagonal boundary terms. In this F-basis, the two sets of pseudo-particle creation operators $\mathcal{T}_{F}^{ \pm}$given by (4.22) and (4.23) take completely symmetric and polarization free forms simultaneously. Moreover, the corresponding two sets of Bethe states become completely symmetric.

\section{$5 \quad$ F-basis}

In this section, we construct the Drinfeld twist [3] (factorizing F-matrix) on the $N$-fold tensor product space $V^{\otimes N}$ (i.e. the quantum space of the open XXZ chain) and the associated representations of the pseudo-particle creation operators in this basis.

\subsection{Factorizing Drinfeld twist $F$}

Let $\mathcal{S}_{N}$ be the permutation group over indices $1, \ldots, N$ and $\left\{\sigma_{i} \mid i=1, \ldots, N-1\right\}$ be the set

of elementary permutations in $\mathcal{S}_{N}$. For each elementary permutation $\sigma_{i}$, we introduce the associated operator $R_{1 \ldots N}^{\sigma_{i}}$ on the quantum space

$$
R_{1 \ldots N}^{\sigma_{i}}(l) \equiv R^{\sigma_{i}}(l)=R_{i, i+1}\left(z_{i}-z_{i+1} \mid l-\eta \sum_{k=1}^{i-1} h^{(k)}\right)
$$

where $l$ is a generic vector in $V$. For any $\sigma, \sigma^{\prime} \in \mathcal{S}_{N}$, operator $R_{1 \ldots N}^{\sigma \sigma^{\prime}}$ associated with $\sigma \sigma^{\prime}$ satisfies the following composition law [2, 6, 7, 10]:

$$
R_{1 \ldots N}^{\sigma \sigma^{\prime}}(l)=R_{\sigma(1 \ldots N)}^{\sigma^{\prime}}(l) R_{1 \ldots N}^{\sigma}(l)
$$


Let $\sigma$ be decomposed in a minimal way in terms of elementary permutations,

$$
\sigma=\sigma_{\beta_{1}} \ldots \sigma_{\beta_{p}}
$$

where $\beta_{i}=1, \ldots, N-1$ and the positive integer $p$ is the length of $\sigma$. The composition law (5.2) enables one to obtain operator $R_{1 \ldots N}^{\sigma}$ associated with each $\sigma \in \mathcal{S}_{N}$. The dynamical quantum Yang-Baxter equation (2.19), weight conservation condition (2.21) and unitary condition (2.22) guarantee the uniqueness of $R_{1 \ldots N}^{\sigma}$. Moreover, one may check that $R_{1 \ldots N}^{\sigma}$ satisfies the following exchange relation with the face type one-row monodromy matrix (4.1)

$$
R_{1 \ldots N}^{\sigma}(l) T_{0,1 \ldots N}^{F}(l \mid u)=T_{0, \sigma(1 \ldots N)}^{F}(l \mid u) R_{1 \ldots N}^{\sigma}\left(l-\eta h^{(0)}\right), \quad \forall \sigma \in \mathcal{S}_{N} .
$$

Now, we construct the face-type Drinfeld twist $F_{1 \ldots N}(l) \equiv F_{1 \ldots N}\left(l ; z_{1}, \ldots, z_{N}\right) 2$ on the $N$-fold tensor product space $V^{\otimes N}$, which satisfies the following three properties [6, 10]:

$$
\begin{aligned}
& \text { I. lower - triangularity; } \\
& \text { II. non - degeneracy; } \\
& \text { III. factorizing property : } R_{1 \ldots N}^{\sigma}(l)=F_{\sigma(1 \ldots N)}^{-1}(l) F_{1 \ldots N}(l), \forall \sigma \in \mathcal{S}_{N} \text {. }
\end{aligned}
$$

Substituting (5.7) into the exchange relation (5.4), we have

$$
F_{\sigma(1 \ldots N)}^{-1}(l) F_{1 \ldots N}(l) T_{0,1 \ldots N}^{F}(l \mid u)=T_{0, \sigma(1 \ldots N)}^{F}(l \mid u) F_{\sigma(1 \ldots N)}^{-1}\left(l-\eta h^{(0)}\right) F_{1 \ldots N}\left(l-\eta h^{(0)}\right) .
$$

Equivalently,

$$
F_{1 \ldots N}(l) T_{0,1 \ldots N}^{F}(l \mid u) F_{1 \ldots N}^{-1}\left(l-\eta h^{(0)}\right)=F_{\sigma(1 \ldots N)}(l) T_{0, \sigma(1 \ldots N)}^{F}(l \mid u) F_{\sigma(1 \ldots N)}^{-1}\left(l-\eta h^{(0)}\right) .
$$

Let us introduce the twisted monodromy matrix $\tilde{T}_{0,1 \ldots N}^{F}(l \mid u)$ by

$$
\begin{aligned}
\tilde{T}_{0,1 \ldots N}^{F}(l \mid u) & =F_{1 \ldots N}(l) T_{0,1 \ldots N}^{F}(l \mid u) F_{1 \ldots N}^{-1}\left(l-\eta h^{(0)}\right) \\
& =\left(\begin{array}{cc}
\tilde{T}_{F}(l \mid u)_{1}^{1} & \tilde{T}_{F}(l \mid u)_{2}^{1} \\
\tilde{T}_{F}(l \mid u)_{1}^{2} & \tilde{T}_{F}(l \mid u)_{2}^{2}
\end{array}\right) .
\end{aligned}
$$

Then (5.9) implies that the twisted monodromy matrix is symmetric under $\mathcal{S}_{N}$, namely,

$$
\tilde{T}_{0,1 \ldots N}^{F}(l \mid u)=\tilde{T}_{0, \sigma(1 \ldots N)}^{F}(l \mid u), \quad \forall \sigma \in \mathcal{S}_{N}
$$

\footnotetext{
${ }^{2}$ In this paper, we adopt the convention: $F_{\sigma(1 \ldots N)}(l) \equiv F_{\sigma(1 \ldots N)}\left(l ; z_{\sigma(1)}, \ldots, z_{\sigma(N)}\right)$.
} 
Define the F-matrix:

$$
F_{1 \ldots N}(l)=\sum_{\sigma \in \mathcal{S}_{N}} \sum_{\left\{\alpha_{j}\right\}=1}^{2} * \prod_{i=1}^{N} P_{\alpha_{\sigma(i)}}^{\sigma(i)} R_{1 \ldots N}^{\sigma}(l),
$$

where $P_{\alpha}^{i}$ is the embedding of the project operator $P_{\alpha}$ in the $i^{\text {th }}$ space with matric elements $\left(P_{\alpha}\right)_{k l}=\delta_{k l} \delta_{k \alpha}$. The sum $\sum^{*}$ in (5.12) is over all non-decreasing sequences of the labels $\alpha_{\sigma(i)}$ :

$$
\begin{array}{ll}
\alpha_{\sigma(i+1)} \geq \alpha_{\sigma(i)} \quad \text { if } \quad & \sigma(i+1)>\sigma(i) \\
\alpha_{\sigma(i+1)}>\alpha_{\sigma(i)} & \text { if } \quad
\end{array}
$$

From (5.13), $F_{1 \ldots N}(l)$ obviously is a lower-triangular matrix. Moreover, the F-matrix is non-

degenerate because all its diagonal elements are non-zero. Using the method of [10], we find that the F-matrix also satisfies the factorizing property (5.7). Hence, the F-matrix $F_{1 \ldots N}(l)$ given by (15.12) is the desirable Drinfeld twist.

\subsection{Completely symmetric representations}

Having found the F-matrix (5.12), let us compute the twisted operators $\tilde{T}_{F}(l \mid u)_{i}^{j}$ defined by (5.10). Using the method similar to that in [10], after a tedious calculation, we obtain the expressions of $\tilde{T}_{F}(l \mid u)_{i}^{j}$. Here we present the results which are relevant,

$$
\begin{aligned}
\tilde{T}_{F}(l \mid u)_{2}^{2}= & \frac{\sin \left(l_{21}-\eta\right)}{\sin \left(l_{21}-\eta+\eta\left\langle H, \epsilon_{1}\right\rangle\right)} \otimes_{i}\left(\begin{array}{cc}
\frac{\sin \left(u-z_{i}\right)}{\sin \left(u-z_{i}+\eta\right)} & \\
1
\end{array}\right)_{(i)} \\
\tilde{T}_{F}(l \mid u)_{1}^{2}= & \sum_{i=1}^{N} \frac{\sin \eta \sin \left(u-z_{i}+l_{12}\right)}{\sin \left(u-z_{i}+\eta\right) \sin l_{12}} E_{12}^{i} \otimes_{j \neq i}\left(\begin{array}{cc}
\frac{\sin \left(u-z_{j}\right) \sin \left(z_{i}-z_{j}+\eta\right)}{\sin \left(u-z_{j}+\eta\right) \sin \left(z_{i}-z_{j}\right)} & \\
1
\end{array}\right)_{(j)} \\
\tilde{T}_{F}(l \mid u)_{2}^{1}= & \frac{\sin \left(l_{21}-\eta\right)}{\sin \left(l_{21}+\eta\left\langle H, \epsilon_{1}-\epsilon_{2}\right\rangle\right)} \sum_{i=1}^{N} \frac{\sin \eta \sin \left(u-z_{i}+l_{21}+\eta+\eta\left\langle H, \epsilon_{1}-\epsilon_{2}\right\rangle\right)}{\sin \left(u-z_{i}+\eta\right) \sin \left(l_{21}+\eta+\eta\left\langle H, \epsilon_{1}-\epsilon_{2}\right\rangle\right)} \\
& \times E_{21}^{i} \otimes_{j \neq i}\left(\begin{array}{cc}
\frac{\sin \left(u-z_{j}\right)}{\sin \left(u-z_{j}+\eta\right)} & \frac{\sin \left(z_{j}-z_{i}+\eta\right)}{\sin \left(z_{j}-z_{i}\right)}
\end{array}\right)_{(j)}
\end{aligned}
$$

where $H=\sum_{k=1}^{N} h^{(k)}$. Applying the above operators to the arbitrary state $\left|i_{1}, \ldots, i_{N}\right\rangle$ given by (4.2), we have

$$
\tilde{T}_{F}(m, l \mid u)_{2}^{2}=\frac{\sin \left(l_{21}-\eta\right)}{\sin \left(l_{2}-m_{1}-\eta\right)} \otimes_{i}\left(\begin{array}{ll}
\frac{\sin \left(u-z_{i}\right)}{\sin \left(u-z_{i}+\eta\right)} & \\
& 1
\end{array}\right)_{(i)}
$$




$$
\begin{aligned}
\tilde{T}_{F}(m, l \mid u)_{1}^{2}= & \sum_{i=1}^{N} \frac{\sin \eta \sin \left(u-z_{i}+l_{12}\right)}{\sin \left(u-z_{i}+\eta\right) \sin l_{12}} \\
& \times E_{12}^{i} \otimes_{j \neq i}\left(\begin{array}{cc}
\frac{\sin \left(u-z_{j}\right) \sin \left(z_{i}-z_{j}+\eta\right)}{\sin \left(u-z_{j}+\eta\right) \sin \left(z_{i}-z_{j}\right)} & \\
1
\end{array}\right)_{(j)}, \\
\tilde{T}_{F}(m, l \mid u)_{2}^{1}= & \frac{\sin \left(l_{21}-\eta\right)}{\sin \left(m_{21}-2 \eta\right)} \sum_{i=1}^{N} \frac{\sin \eta \sin \left(u-z_{i}+m_{21}-\eta\right)}{\sin \left(u-z_{i}+\eta\right) \sin \left(m_{21}-\eta\right)} \\
& \times E_{21}^{i} \otimes_{j \neq i}\left(\begin{array}{cc}
\frac{\sin \left(u-z_{j}\right)}{\sin \left(u-z_{j}+\eta\right)} & \frac{\sin \left(z_{j}-z_{i}+\eta\right)}{\sin \left(z_{j}-z_{i}\right)}
\end{array}\right)_{(j)} .
\end{aligned}
$$

It then follows that the two pseudo-particle creation operators (4.22) and (4.23) in the F-basis simultaneously have the following completely symmetric polarization free forms:

$$
\begin{aligned}
& \tilde{\mathcal{T}}_{F}^{-}(m, \lambda \mid u)_{1}^{2}=\frac{\sin m_{12}}{\sin \left(m_{1}-\lambda_{2}\right)} \prod_{k=1}^{N} \frac{\sin \left(u+z_{k}\right)}{\sin \left(u+z_{k}+\eta\right)} \\
& \times \sum_{i=1}^{N} \frac{\sin \left(\lambda_{1}+\xi-z_{i}\right) \sin \left(\lambda_{2}+\xi+z_{i}\right) \sin 2 u \sin \eta}{\sin \left(\lambda_{1}+\xi+u\right) \sin \left(\lambda_{2}+\xi+u\right) \sin \left(u-z_{i}+\eta\right) \sin \left(u+z_{i}\right)} \\
& \times E_{12}^{i} \otimes_{j \neq i}\left(\begin{array}{cc}
\frac{\sin \left(u-z_{j}\right) \sin \left(u+z_{j}+\eta\right) \sin \left(z_{i}-z_{j}+\eta\right)}{\sin \left(u-z_{j}+\eta\right) \sin \left(u+z_{j}\right) \sin \left(z_{i}-z_{j}\right)} & \\
& 1
\end{array}\right)_{(j)}, \\
& \tilde{\mathcal{T}}_{F}^{+}(\lambda, m \mid u)_{2}^{1}=\frac{\sin \left(m_{21}+\eta\right)}{\sin \left(m_{2}-\lambda_{1}\right)} \prod_{k=1}^{N} \frac{\sin \left(u+z_{k}\right)}{\sin \left(u+z_{k}+\eta\right)} \\
& \times \sum_{i=1}^{N} \frac{\sin \left(\lambda_{2}+\bar{\xi}-z_{i}\right) \sin \left(\lambda_{1}+\bar{\xi}+z_{i}\right) \sin (2 u+2 \eta) \sin \eta}{\sin \left(\lambda_{1}+\bar{\xi}-u-\eta\right) \sin \left(\lambda_{2}+\bar{\xi}-u-\eta\right) \sin \left(u+z_{i}\right) \sin \left(u-z_{i}+\eta\right)} \\
& \times E_{21}^{i} \otimes_{j \neq i}\left(\begin{array}{ll}
\frac{\sin \left(u-z_{j}\right) \sin \left(u+z_{j}+\eta\right)}{\sin \left(u-z_{j}+\eta\right) \sin \left(u+z_{j}\right)} & \\
& \frac{\sin \left(z_{j}-z_{i}+\eta\right)}{\sin \left(z_{j}-z_{i}\right)}
\end{array}\right)_{(j)} .
\end{aligned}
$$

Now let us evaluate the two sets of Bethe states (4.24) and (4.25) in the F-basis (or the twisted Bethe states)

$$
\begin{aligned}
{\overline{\left|v_{1}^{(1)}, \cdots, v_{M}^{(1)}\right\rangle_{F}^{(I)}}}^{(I)} F_{1 \ldots N}(\lambda)\left|v_{1}^{(1)}, \cdots, v_{M}^{(1)}\right\rangle_{F}^{(I)} \\
\frac{\left|v_{1}^{(2)}, \cdots, v_{M}^{(2)}\right\rangle_{F}^{(I I)}}{}=F_{1 \ldots N}(\lambda)\left|v_{1}^{(2)}, \cdots, v_{M}^{(2)}\right\rangle_{F}^{(I I)}
\end{aligned}
$$

Since $|1, \ldots, 1\rangle$ and $|2, \ldots, 2\rangle$ are invariant under the action of the F-matrix $F_{1 \ldots N}(l)(\underline{5.12})$, namely,

$$
F_{1 \ldots N}(l)|i, \ldots, i\rangle=|i, \ldots, i\rangle, \quad i=1,2,
$$


we have

$$
\begin{aligned}
& \overline{\left|v_{1}^{(1)}, \cdots, v_{M}^{(1)}\right\rangle_{F}^{(I)}}=\tilde{\mathcal{T}}_{F}^{+}\left(\lambda, \lambda+2 \eta \hat{1} \mid v_{1}^{(1)}\right)_{2}^{1} \ldots \tilde{\mathcal{T}}_{F}^{+}\left(\lambda, \lambda+2 M \eta \hat{1} \mid v_{M}^{(1)}\right)_{2}^{1}|1, \ldots, 1\rangle, \\
& \overline{\left|v_{1}^{(2)}, \cdots, v_{M}^{(2)}\right\rangle_{F}^{(I I)}}=\tilde{\mathcal{T}}_{F}^{-}\left(\lambda-2 \eta \hat{2}, \lambda \mid v_{1}^{(2)}\right)_{1}^{2} \cdots \tilde{\mathcal{T}}_{F}^{-}\left(\lambda-2 M \eta \hat{2}, \lambda \mid v_{M}^{(2)}\right)_{1}^{2}|2, \ldots, 2\rangle .
\end{aligned}
$$

Thanks to the polarization free representations (5.20) and (5.21) of the pseudo-particle creation operators, we also obtain completely symmetric expressions of the two sets of Bethe sates in the F-basis:

$$
\begin{aligned}
& \overline{\left|v_{1}^{(1)}, \cdots, v_{M}^{(1)}\right\rangle_{F}^{(I)}}=\prod_{k=1}^{M}\left\{\frac{\sin \left(\lambda_{12}-\eta+2 k \eta\right)}{\sin \left(\lambda_{12}+k \eta\right)} \prod_{n=1}^{N} \frac{\sin \left(v_{k}^{(1)}-z_{n}\right)}{\sin \left(v_{k}^{(1)}-z_{n}+\eta\right)}\right\} \\
& \times \sum_{i_{1}<i_{2} \ldots<i_{M}} B_{M}^{(I)}\left(\left\{v_{\alpha}^{(1)}\right\} \mid\left\{z_{i_{n}}\right\}\right) E_{21}^{i_{1}} \ldots E_{21}^{i_{M}}|1, \ldots, 1\rangle, \\
& \overline{\left|v_{1}^{(2)}, \cdots, v_{M}^{(2)}\right\rangle_{F}^{(I I)}}=\prod_{k=1}^{M}\left\{\frac{\sin \left(\lambda_{12}+2 k \eta\right)}{\sin \left(\lambda_{12}+k \eta\right)} \prod_{n=1}^{N} \frac{\sin \left(v_{k}^{(2)}+z_{n}\right)}{\sin \left(v_{k}^{(2)}+z_{n}+\eta\right)}\right\} \\
& \times \sum_{i_{1}<i_{2} \ldots<i_{M}} B_{M}^{(I I)}\left(\left\{v_{\alpha}^{(2)}\right\} \mid\left\{z_{i_{n}}\right\}\right) E_{12}^{i_{1}} \ldots E_{12}^{i_{M}}|2, \ldots, 2\rangle .
\end{aligned}
$$

Here the functions $B_{M}^{(I)}\left(\left\{v_{\alpha}\right\} \mid\left\{z_{i_{n}}\right\}\right)$ and $B_{M}^{(I I)}\left(\left\{v_{\alpha}\right\} \mid\left\{z_{i_{n}}\right\}\right)$ are given by

$$
\begin{aligned}
& B_{M}^{(I)}\left(\left\{v_{\alpha}\right\} \mid\left\{z_{i_{n}}\right\}\right)=\prod_{n=1}^{M} \prod_{k=1}^{M} \frac{\sin \left(v_{n}-z_{i_{k}}+\eta\right) \sin \left(v_{n}+z_{i_{k}}\right)}{\sin \left(v_{n}-z_{i_{k}}\right) \sin \left(v_{n}+z_{i_{k}}+\eta\right)} \\
& \quad \times \sum_{\sigma \in \mathcal{S}_{M}} \prod_{n=1}^{M}\left\{\frac{\sin \left(\lambda_{2}+\bar{\xi}-z_{i_{\sigma(n)}}\right) \sin \left(\lambda_{1}+\bar{\xi}+z_{i_{\sigma(n)}}\right) \sin \left(2 v_{n}+2 \eta\right) \sin \eta}{\sin \left(\lambda_{2}+\bar{\xi}-v_{n}-\eta\right) \sin \left(\lambda_{1}+\bar{\xi}-v_{n}-\eta\right) \sin \left(v_{n}+z_{i_{\sigma(n)}}\right) \sin \left(v_{n}-z_{i_{\sigma(n)}}+\eta\right)}\right. \\
& \left.\quad \times \prod_{k>n}^{M} \frac{\sin \left(v_{k}-z_{i_{\sigma(n)}}\right) \sin \left(v_{k}+z_{i_{\sigma(n)}}+\eta\right) \sin \left(z_{i_{\sigma(k)}}-z_{i_{\sigma(n)}}+\eta\right)}{\sin \left(v_{k}-z_{i_{\sigma(n)}}+\eta\right) \sin \left(v_{k}+z_{i_{\sigma(n)}}\right) \sin \left(z_{i_{\sigma(k)}}-z_{i_{\sigma(n)}}\right)}\right\}, \\
& B_{M}^{(I I)}\left(\left\{v_{\alpha}\right\} \mid\left\{z_{i_{n}}\right\}\right)=\sum_{\sigma \in \mathcal{S}_{M}} \prod_{n=1}^{M}\left\{\frac{\sin \left(\lambda_{1}+\xi-z_{i_{\sigma(n)}}\right) \sin \left(\lambda_{2}+\xi+z_{i_{\sigma(n)}}\right) \sin \left(2 v_{n}\right) \sin \eta}{\sin \left(\lambda_{1}+\xi+v_{n}\right) \sin \left(\lambda_{2}+\xi+v_{n}\right) \sin \left(v_{n}-z_{i_{\sigma(n)}}+\eta\right) \sin \left(v_{n}+z_{i_{\sigma(n)}}\right)}\right. \\
& \left.\quad \times \prod_{k>n}^{M} \frac{\sin \left(v_{n}-z_{\left.i_{\sigma(k)}\right)}\right) \sin \left(v_{n}+z_{i_{\sigma(k)}}+\eta\right) \sin \left(z_{i_{\sigma(n)}}-z_{i_{\sigma(k)}}+\eta\right)}{\sin \left(v_{n}-z_{i_{\sigma(k)}}+\eta\right) \sin \left(v_{n}+z_{\sigma_{\sigma(k)}}\right) \sin \left(z_{i_{\sigma(n)}}-z_{i_{\sigma(k)}}\right)}\right\} .
\end{aligned}
$$

We remark that if the parameters $\left\{v_{k}^{(1)}\right\}$ (or $\left\{v_{k}^{(2)}\right\}$ ) do not satisfy the associated Bethe ansatz equations (2.45) (or (2.47)), the corresponding twisted states (5.25) and (5.26) become offshell Bethe states. These off-shell Bethe states can still be expressed in the same forms as those of (5.27)-(5.30) (but the corresponding parameters are not necessarily the roots of the Bethe ansatz equations). 


\section{Conclusions}

We have constructed the factorizing F-matrix (5.12) in the face picture for the open XXZ chain with non-diagonal boundary terms, where the non-diagonal K-matrices $K^{ \pm}(u)$ are given by (2.9) and (2.11). It is found that in the F-basis the pseudo-particle creation operators, which generate the complete set of the eigenstates of the model, simultaneously take the completely symmetric and polarization free forms (5.20) and (5.21). This allows us to obtain the explicit and completely symmetric expressions (5.27) and (5.28) of the two sets of (off-shell) Bethe states.

The results of this paper make it feasible to derive the determinant representations of partition functions and scalar products of the complete Bethe states for the open XXZ chain with non-diagonal boundary terms specified by the non-diagonal K-matrices $K^{ \pm}(u)(2.9)$ and (2.11). These results will be presented elsewhere [34].

\section{Acknowledgements}

The financial support from Australian Research Council is gratefully acknowledged.

\section{Appendix A: Bethe states (3.3)}

In this appendix, we will prove that if the parameters $\left\{v_{k}^{(1)}\right\}$ satisfy the first set of Bethe ansatz equations (2.45) the Bethe states (3.3) give rise to the eigenvalues (2.46) of the transfer matrix.

Using the QYBE (2.2), the dual RE (2.5) and the definition of the monodromy matrix $\mathbb{T}^{+}(u)(3.2)$, we can show that $\mathbb{T}^{+}(u)$ satisfies the following relation

$$
\begin{aligned}
\bar{R}_{2,1}\left(u_{2}-u_{1}\right)\left(\mathbb{T}_{1}^{+}\left(u_{1}\right)\right)^{t_{1}} \bar{R}_{1,2}\left(-u_{1}-u_{2}-2 \eta\right)\left(\mathbb{T}_{2}^{+}\left(u_{2}\right)\right)^{t_{2}} \\
=\left(\mathbb{T}_{2}^{+}\left(u_{2}\right)\right)^{t_{2}} \bar{R}_{2,1}\left(-u_{1}-u_{2}-2 \eta\right)\left(\mathbb{T}_{1}^{+}\left(u_{1}\right)\right)^{t_{1}} \bar{R}_{1,2}\left(u_{2}-u_{1}\right) .
\end{aligned}
$$

This, together with (2.27) and (2.33)-(2.34), implies that the operators $\mathcal{T}^{+}(m \mid u)$ defined by (3.1) satisfy the relations

$$
\begin{array}{rl}
\sum_{i_{1}, j_{1}} \sum_{i_{2}, j_{2}} R & R\left(u_{2}-u_{1} ; m\right)_{i_{1} j_{1}}^{i_{0} j_{0}} R\left(-u_{1}-u_{2}-2 \eta ; m\right)_{j_{2} i_{3}}^{j_{1} i_{2}} \\
\times & \mathcal{T}^{+}\left(m+\eta\left(\hat{\imath}_{3}+\hat{\jmath}_{2}\right) \mid u_{2}\right)_{j_{3}}^{j_{2}} \mathcal{T}^{+}\left(m+\eta\left(\hat{\imath}_{1}+\hat{\jmath}_{1}\right) \mid u_{1}\right)_{i_{2}}^{i_{1}}
\end{array}
$$




$$
\begin{aligned}
& =\sum_{i_{1}, j_{1}} \sum_{i_{2}, j_{2}} R\left(-u_{1}-u_{2}-2 \eta ; m\right)_{i_{1} j_{2}}^{i_{0} j_{1}} R\left(u_{2}-u_{1} ; m\right)_{j_{3} i_{3}}^{j_{2} i_{2}} \\
& \quad \times \mathcal{T}^{+}\left(m+\eta\left(\hat{\imath}_{1}+\hat{\jmath}_{2}\right) \mid u_{1}\right)_{i_{2}}^{i_{1}} \mathcal{T}^{+}\left(m+\eta\left(\hat{\imath}_{0}+\hat{\jmath}_{0}\right) \mid u_{2}\right)_{j_{1}}^{j_{0}} .
\end{aligned}
$$

Following [28], let us introduce operators $\mathcal{A}^{+(1)}$ and $\mathcal{D}^{+(1)}$

$$
\begin{aligned}
& \mathcal{D}^{+(1)}(m \mid u)=\mathcal{T}^{+}(m \mid u)_{2}^{2}, \\
& \mathcal{A}^{+(1)}(m \mid u)=\frac{\sin \left(m_{12}-\eta\right)}{\sin m_{12}}\left\{\mathcal{T}^{+}(m \mid u)_{1}^{1}-R(-2 u-2 \eta ; m+\eta \hat{2})_{21}^{12} \mathcal{T}^{+}(m \mid u)_{2}^{2}\right\}
\end{aligned}
$$

We may check that the transfer matrix $\tau(u)(2.7)$ can be expressed in terms of a linear combination of the above operators

$$
\begin{aligned}
\tau(u)= & \operatorname{tr}\left(K^{+}(u) \mathbb{T}(u)\right)=\operatorname{tr}\left(\left(K^{-}(u)\right)^{t_{0}}\left(\mathbb{T}^{+}(u)\right)^{t_{0}}\right)=\operatorname{tr}\left(K^{-}(u) \mathbb{T}^{+}(u)\right) \\
= & \frac{\sin \left(\lambda_{1}+\xi-u\right)}{\sin \left(\lambda_{1}+\xi+u\right)} \mathcal{A}^{+(1)}(\lambda \mid u) \\
& \quad+\frac{\sin \left(\lambda_{1}+\xi+u+\eta\right) \sin \left(\lambda_{2}+\xi-u-\eta\right) \sin (2 u)}{\sin \left(\lambda_{1}+\xi+u\right) \sin \left(\lambda_{2}+\xi+u\right) \sin (2 u+\eta)} \mathcal{D}^{+(1)}(\lambda \mid u) .
\end{aligned}
$$

Using the technique developed in [16], after tedous calculations, we find that the state $\left|\Omega^{(I)}(\lambda)\right\rangle$ given by (3.4) is a reference state in the usual sense,

$$
\begin{aligned}
\mathcal{A}^{+(1)}(\lambda+N \eta \hat{1} \mid u)\left|\Omega^{(I)}(\lambda)\right\rangle= & \frac{\sin \left(\lambda_{2}+\bar{\xi}-u\right) \sin \left(\lambda_{1}+\bar{\xi}+u\right)}{\sin \left(\lambda_{2}+\bar{\xi}-u-\eta\right) \sin \left(\lambda_{1}+\bar{\xi}-u-\eta\right)} \\
& \times \frac{\sin (2 u+2 \eta)}{\sin (2 u+\eta)}\left|\Omega^{(I)}(\lambda)\right\rangle, \\
\mathcal{D}^{+(1)}(\lambda+N \eta \hat{1} \mid u)\left|\Omega^{(I)}(\lambda)\right\rangle= & \prod_{l=1}^{N} \frac{\sin \left(u+z_{l}\right) \sin \left(u-z_{l}\right)}{\sin \left(u+z_{l}+\eta\right) \sin \left(u-z_{l}+\eta\right)} \\
& \times \frac{\sin \left(\lambda_{2}+\bar{\xi}+u+\eta\right)}{\sin \left(\lambda_{2}+\bar{\xi}-u-\eta\right)}\left|\Omega^{(I)}(\lambda)\right\rangle, \\
\mathcal{T}^{+}(\lambda+N \eta \hat{1} \mid u)_{2}^{1}\left|\Omega^{(I)}(\lambda)\right\rangle \neq & 0, \\
\mathcal{T}^{+}(\lambda+N \eta \hat{1} \mid u)_{1}^{2}\left|\Omega^{(I)}(\lambda)\right\rangle= & 0 .
\end{aligned}
$$

Moreover, by (A.1) we obtain the commutation relations,

$$
\begin{aligned}
\mathcal{A}^{+(1)}\left(m+\eta(\hat{1}+\hat{2}) \mid u_{1}\right) \mathcal{T}^{+}\left(m+2 \eta \hat{1} \mid u_{2}\right)_{2}^{1} \\
=\frac{\sin \left(u_{2}-u_{1}+\eta\right) \sin \left(u_{2}+u_{1}\right)}{\sin \left(u_{2}-u_{1}\right) \sin \left(u_{2}+u_{1}+\eta\right)} \mathcal{T}^{+}\left(m+2 \eta \hat{1} \mid u_{2}\right){ }_{2}^{1} \mathcal{A}^{+(1)}\left(m+2 \eta \hat{1} \mid u_{1}\right) \\
\quad-\frac{\sin (\eta) \sin \left(2 u_{1}\right) \sin \left(u_{2}-u_{1}+\lambda_{21}-\eta\right)}{\sin \left(u_{2}-u_{1}\right) \sin \left(2 u_{1}+\eta\right) \sin \left(\lambda_{21}-\eta\right)} \mathcal{T}^{+}\left(m+2 \eta \hat{1} \mid u_{1}\right){ }_{2}^{1} \mathcal{A}^{+(1)}\left(m+2 \eta \hat{1} \mid u_{2}\right)
\end{aligned}
$$




$$
\begin{gathered}
-\frac{\sin (\eta) \sin \left(2 u_{2}+2 \eta\right) \sin \left(2 u_{1}\right) \sin \left(u_{2}-u_{1}+\lambda_{21}\right)}{\sin \left(u_{1}+u_{2}+\eta\right) \sin \left(2 u_{2}+\eta\right) \sin \left(2 u_{1}+\eta\right) \sin \left(\lambda_{21}-\eta\right)} \\
\quad \times \mathcal{T}^{+}\left(m+2 \eta \hat{1} \mid u_{1}\right){ }_{2}^{1} \mathcal{D}^{+(1)}\left(m+2 \eta \hat{1} \mid u_{2}\right), \\
\mathcal{D}^{+(1)}\left(m+2 \eta \hat{2} \mid u_{2}\right) \mathcal{T}^{+}\left(m+\eta(\hat{1}+\hat{2}) \mid u_{1}\right){ }_{2}^{1} \\
=\frac{\sin \left(u_{2}+u_{1}+2 \eta\right) \sin \left(u_{2}-u_{1}+\eta\right)}{\sin \left(u_{2}+u_{1}+\eta\right) \sin \left(u_{2}-u_{1}\right)} \mathcal{T}^{+}\left(m+\eta(\hat{1}+\hat{2}) \mid u_{1}\right){ }_{2}^{1} \mathcal{D}^{+(1)}\left(m+\eta(\hat{1}+\hat{2}) \mid u_{2}\right) \\
-\frac{\sin (\eta) \sin \left(2 u_{1}+2 \eta\right) \sin \left(u_{2}-u_{1}+\lambda_{21}+\eta\right)}{\sin \left(u_{2}-u_{1}\right) \sin \left(2 u_{1}+\eta\right) \sin \left(\lambda_{21}+\eta\right)} \\
\quad \times \mathcal{T}^{+}\left(m+\eta(\hat{1}+\hat{2}) \mid u_{2}\right){ }_{2}^{1} \mathcal{D}^{+(1)}\left(m+\eta(\hat{1}+\hat{2}) \mid u_{1}\right) \\
-\frac{\sin (\eta) \sin \left(u_{2}+u_{1}+\lambda_{21}+2 \eta\right)}{\sin \left(u_{1}+u_{2}+\eta\right) \sin \left(\lambda_{12}-\eta\right)} \\
\quad \times \mathcal{T}^{+}\left(m+\eta(\hat{1}+\hat{2}) \mid u_{2}\right){ }_{2}^{1} \mathcal{A}^{+(1)}\left(m+\eta(\hat{1}+\hat{2}) \mid u_{1}\right) .
\end{gathered}
$$

Carrying out the generalized Bethe ansatz [16], we finally find that the Bethe states $\left|v_{1}^{(1)}, \cdots, v_{M}^{(1)}\right\rangle^{(I)}$ (3.3) give rise to the first set of eigenvalues $\Lambda^{(1)}(u)(2.46)$ provided that the parameters $\left\{v_{k}^{(1)}\right\}$ satisfy the first set of Bethe ansatz equations (2.45).

\section{References}

[1] V. E. Korepin, N. M. Bogoliubov and A. G. Izergin, Quantum Inverse Scattering Method and Correlation Functions, Cambridge University Press, 1993.

[2] J.M. Maillet and J. Sanchez de Santos, Drinfeld twists and algebraic Bethe ansatz, Amer. Math. Soc. Transl. 201 (2000), 137.

[3] V. G. Drinfeld, Sov. Math. Dokl. 28 (1983), 667.

[4] N. Kitanine, J. M. Maillet and V. Terras, Nucl. Phys. B 554 (1999), 647.

[5] V. Terras, Lett. Math. Phys. 48 (1999), 263.

[6] T. -D. Albert, H. Boos, R. Flume and K. Rulig, J. Phys. A 33 (2000), 4963.

[7] T. -D. Albert, H. Boos, R. Flume, R. H. Poghossian and K. Rulig, Lett. Math. Phys. 53 (2000), 201.

[8] T. -D. Albert and K. Rulig, J. Phys. A 34 (2001), 1569. 
[9] S.-Y. Zhao, W.-L. Yang and Y.-Z. Zhang, J. stat. Mech. (2005), P04005; Commun. Math. Phys. 268 (2006), 505; Int. J. Mod. Phys. B 20 (2006), 505.

[10] W.-L. Yang, Y.-Z. Zhang and S.-Y. Zhao, JHEP 12 (2004), 038; Commun. Math. Phys. 264 (2006), 87.

[11] Y.-S. Wang, Nucl. Phys. B 622 (2002), 633.

[12] N. Kitanine, K. K. Kozlowski, J. M. Maillet, G. Niccoli, N. A. Slavnov and V. Terras, J. Stat. Mech. (2007), P10009.

[13] E. K. Sklyanin, J. Phys. A 21 (1988), 2375.

[14] R. I. Nepomechie, J. Stat. Phys. 111 (2003), 1363; J. Phys. A 37 (2004), 433.

[15] J. Cao, H. -Q. Lin, K.-J. Shi and Y. Wang, Nucl. Phys. B 663 (2003), 487.

[16] W.-L. Yang and R. Sasaki, Nucl. Phys. B 679 (2004), 495; J. Math. Phys. 45 (2004), 4301; W.-L. Yang, R. Sasaki and Y.-Z. Zhang, JHEP 09 (2004), 046.

[17] W. Galleas and M. J. Martins, Phys. Lett. A 335 (2005), 167; C.S. Melo, G. A. P. Ribeiro and M. J. Martins, Nucl. Phys. B 711 (2005), 565.

[18] J. de Gier and P. Pyatov, J. Stat. Mech. (2004), P03002; A. Nichols, V. Rittenberg and J. de Gier, J. Stat. Mech. (2005), P05003; J. de Gier, A. Nichols, P. Pyatov and V. Rittenberg, Nucl. Phys. B 729 (2005), 387.

[19] J. de Gier and F. H. L. Essler, Phys. Rev. Lett. 95 (2005), 240601; J. Stat. Mech. (2006), P 12011.

[20] W.-L. Yang, Y.-Z. Zhang and M. Gould, Nucl. Phys. B 698 (2004), 312.

[21] Z. Bajnok, J. Stat. Mech. (2006), P06010.

[22] W.-L. Yang and Y.-Z. Zhang, JHEP 01 (2005), 021; W.-L. Yang, Y.-Z. Zhang and R. Sasaki, Nucl. Phys. 729 (2005), 594.

[23] A. Doikou and P.P. Martin, J. Stat. Mech. (2006), P06004; A. Dikou, J. Stat. Mech. (2006), P09010. 
[24] R. Murgan, R. I. Nepomechie and C. Shi, J. Stat. Mech. (2006) P08006.

[25] P. Baseilhac and K. Koizumi, J. Stat. Mech. (2007), P09006.

[26] W. Galleas, Nucl. Phys. B 790 (2008), 524.

[27] R. Murgan, JHEP 04 (2009), 076.

[28] W.-L. Yang and Y.-Z. Zhang, JHEP 04 (2007), 044; Nucl. Phys. B 789 (2008), 591.

[29] R. I. Nepomechie and F. Ravanini, J. Phys. A 36 (2003), 11391; Addendum, J. Phys. A 37 (2004), 1945.

[30] W.-L. Yang, R. I. Nepomechie and Y.-Z. Zhang, Phys. Lett. B 633 (2006), 664; W.-L. Yang, and Y.-Z. Zhang, Nucl. Phys. B 744 (2006), 312; L. Frappat, R. I. Nepomechie and E. Ragoucy, J. Stat. Mech. (2007), P09008.

[31] H. J. de Vega and A. Gonzalez-Ruiz, J. Phys. A 26 (1993), L519.

[32] S. Ghoshal and A. B. Zamolodchikov, Int. J. Mod. Phys. A 9 (1994), 3841.

[33] R. J. Baxter, Exactly solved models in statistical mechanics, Academic Press, New York, 1982.

[34] W.-L. Yang, X. Chen, J. Feng, K. Hao, B.-Y. Hou, K.-J. Shi and Y.-Z. Zhang, Determinant representations of scalar products for the open XXZ chain with non-diagonal boundary terms.

[35] G. Felder and A. Varchenko, Nucl. Phys. B 480 (1996), 485.

[36] B. Y. Hou, R. Sasaki and W.-L. Yang, Nucl. Phys. B 663 (2003), 467; J. Math. Phys. 45 (2004), 559 . 